\title{
On the Spatial Locality of Electronic Correlations in LiFeAs
}

\author{
Minjae Kim,, , 2, * Hu Miao, ${ }^{3}$ Sangkook Choi, ${ }^{4}$ Manuel Zingl, ${ }^{5}$ Antoine Georges,,${ }^{6,5,7,8}$ and Gabriel Kotliar ${ }^{1,4}$ \\ ${ }^{1}$ Department of Physics and Astronomy, Rutgers University, Piscataway, New Jersey 08854, USA \\ ${ }^{2}$ Department of Chemistry, Pohang University of Science and Technology (POSTECH), Pohang 37673, Korea \\ ${ }^{3}$ Materials Science and Technology Division, Oak Ridge National Laboratory, Oak Ridge, Tennessee 37831, USA \\ ${ }^{4}$ Condensed Matter Physics and Materials Science Department, \\ Brookhaven National Laboratory, Upton, New York 11973, USA \\ ${ }^{5}$ Center for Computational Quantum Physics, Flatiron Institute, 162 5th Avenue, New York, NY 10010, USA \\ ${ }^{6}$ Collège de France, 11 place Marcelin Berthelot, 75005 Paris, France \\ ${ }^{7}$ Centre de Physique Théorique, École Polytechnique, \\ CNRS, Université Paris-Saclay, 91128 Palaiseau, France \\ ${ }^{8}$ Department of Quantum Matter Physics, University of Geneva, \\ 24 Quai Ernest-Ansermet, 1211 Geneva 4, Switzerland
}

(Dated: February 9, 2021)

\begin{abstract}
We address the question of the degree of spatial non-locality of the self energy in the iron-based superconductors, a subject which is receiving considerable attention. Using LiFeAs as a prototypical example, we extract the self energy from angular-resolved photoemission spectroscopy (ARPES) data. We use two distinct electronic structure references: density functional theory in the local density approximation and linearized quasiparticle self consistent GW (LQSGW). We find that with the LQSGW reference, spatially local dynamical correlations provide a consistent description of the experimental data, and account for some surprising aspects of the data such as the substantial out of plane dispersion of the electron Fermi surface having dominant $x z / y z$ character. Hence, correlations effects can be separated into static non-local contributions well described by LQSGW and dynamical local contributions. Hall effect and resistivity data are shown to be consistent with this description.
\end{abstract}

Introduction. The origin of superconductivity in the iron pnictides and chalcogenides is an outstanding open problem in condensed matter physics [1]. Two opposite points of view have been presented. In the first one, superconductivity originates from the exchange of spatially non-local antiferromagnetic (AFM) spin fluctuations [24. and non-local correlations are also essential in the normal state [5 8]. The second one posits a more local pairing due to Hund's coupling [9 13], which in turns requires a rather local picture of the normal state.

Answering this question requires a proper understanding of the degree of spatial locality of electronic correlations in the normal state. This has been addressed previously by a comparison of theoretical calculations to experiments. Some results favor the local picture 14 22. while others support the non local view [5, 6, 23 25].

Here, we take a different approach and address this question by a direct examination of experimental data from angle-resolved photoemission spectroscopy (ARPES), following the approach which was successful for $\mathrm{Sr}_{2} \mathrm{RuO}_{4}$ [26]. We consider LiFeAs [27], a prototypical iron-based superconductor which is free from magnetic and nematic instabilities and which has been intensively studied for more than a decade [7, 14, 18], and use the experimentally measured quasiparticle dispersions for the different Fermi surface (FS) sheets to determine the self-energy and assess its degree of spatial locality.

Our results offer a solution to the local vs. non-local conundrum [28]. We find that the electronic self-energy can be separated, to a good approximation, into a nonlocal part which is frequency independent, and a dynam- ical (frequency-dependent) part which is spatially local to a good approximation. The non-local part can be incorporated in the reference Hamiltonian with respect to which the dynamical self-energy is defined, and we show that the quasiparticle GW approximation [29 31] provides a good starting point to that effect. These findings are in line with previous work by Tomczak et al. 22, 29], but we emphasize that our conclusions are established directly from experimental observations, once the proper reference Hamiltonian to define self energies is used. This finding rationalizes the success of dynamical mean field theory (DMFT) [32, 33] for these materials [14, 34, 35], and emphasizes GW+DMFT as a method of choice in this context [22, 29, 36].

Method. Ignoring photoemission matrix elements, extrinsic and surface effects, we relate the measured photoemission spectra to the spectral function associated with the one particle Green's function:

$$
G(k, \omega)=[\omega \cdot \mathbf{I}-H(k)-\Sigma(k, \omega)]_{m \sigma, m^{\prime} \sigma^{\prime}}^{-1}
$$

In this expression, $H(k)$ is a reference Hamiltonian matrix expressed in a localised basis of orbitals $m, \sigma$ ( $\sigma$ is the spin index), $\omega$ is the frequency, and $k$ is the wavevector in the Brillouin zone. $\Sigma(k, \omega)$ is the self-energy matrix for the given reference Hamiltonian $H(k)$. The chemical potential is included in $H(k)$.

We consider two different choices for the reference Hamiltonian $H(k)$. The first is the Kohn-Sham Hamiltonian obtained from density-functional theory in the local density approximation (DFT-LDA) using the Wien2k software package [37, 38]. The second is the quasiparti- 
Table I. The net Fermi surface volumes, $\mathrm{V}_{\mathrm{FS} \text {,total }}^{\text {electron }} \mathrm{V}_{\mathrm{FS} \text {,total }}^{\text {hole }}$ and Fermi surface volumes of each sheet (electrons/unit cell) in (a) the LDA, (b) the LDA+non-local $\Sigma$ ansatz, $\left(k_{z}=0.00\right.$ for fitting of hole bands and $k_{z}=0.35$ for fitting of electron bands), (c) the LDA+local $\Sigma$ ansatz, $\left(k_{z}=0.00\right.$ for fitting of hole bands and $k_{z}=0.35$ for fitting of electron bands), (d) the LQSGW, (e) the LQSGW+non-local $\Sigma$ ansatz, $\left(k_{z}=0.00\right.$ for fitting of hole bands and $k_{z}=0.55$ for fitting of electron bands). 0.02-0.03 (electrons/unit cell) in the net Fermi surface volume is the numerical uncertainty.

\begin{tabular}{||l||l|l|l|l|l|l||}
\hline & $\alpha^{\prime}$ & $\alpha$ & $\beta$ & $\gamma$ & $\delta$ & Net \\
\hline LDA & 0.01 & 0.14 & 0.33 & 0.18 & 0.28 & -0.02 \\
\hline LDA+non-local $\Sigma$ & 0.00 & 0.08 & 0.37 & 0.23 & 0.39 & +0.17 \\
\hline LDA+local $\Sigma$ & 0.00 & 0.06 & 0.36 & 0.19 & 0.35 & +0.12 \\
\hline LQSGW & 0.00 & 0.08 & 0.35 & 0.20 & 0.21 & -0.03 \\
\hline LQSGW+non-local $\Sigma$ & 0.00 & 0.05 & 0.36 & 0.20 & 0.26 & +0.04 \\
\hline
\end{tabular}

cle Hamiltonian obtained from the linearized quasiparticle self-consistent GW method (LQSGW) using the FlapwMBPT code [30, 31]. For the localised basis set $(m \sigma)$, we calculate maximally localized Wannier functions 39, 40 in a wide energy window including $\mathrm{Fe}(d)$ and $\operatorname{As}(p)$ orbitals, using the Wannier90 [41], Wien2Wannier [42] and ComDMFT 36] packages. (See the Supplemental Material (SM) [43].) We take the spin-orbit coupling (SOC) to be local and present only on iron atoms (see SM [43]).

We first discuss the electronic structure associated with $H(k)$, i.e. in the absence of the self-energy. In Fig. 1(a), we compare the FS of DFT-LDA to that of LQSGW. The LQSGW FS clearly displays a significant shrinking of the $x z / y z$ dominated hole/electron pockets, $\alpha^{\prime}, \alpha$, and $\delta$ sheets in comparison to LDA, as pointed out in previous work [5, 22, 29]. This is because non-local electronic interactions are more prominently taken into account in the LQSGW, resulting in a repulsion of the bands between $\alpha\left(\alpha^{\prime}\right)$ and $\delta$. The shrinking of these FS pockets from LDA to LQSGW is also apparent from Table I] in which we compare the volumes of the different FS sheets between the two methods. The net difference between all electron and hole FS volumes is also indicated and, for both methods, adds up to zero within error bars as required by the Luttinger's theorem.

The procedure for extracting the self-energy from ARPES data follows Ref. 26] for $\mathrm{Sr}_{2} \mathrm{RuO}_{4}$. From a theoretical viewpoint, the dispersions of the different branches of quasiparticles are the solutions of $\operatorname{det}[\omega-$ $H(k)-\operatorname{Re} \Sigma(k, \omega)]=0$ (neglecting the lifetime effects associated with $\operatorname{Im} \Sigma$ ). We use the measured positions of the maximum of the momentum distribution curves (MDC) associated with several quasiparticle bands, for a given binding energy $\omega$, as an input to this equation which is then solved by a numerical root-finding procedure for the real part of the self-energy (for details of the procedure, see SM [43]).

To facilitate the determination of $\Sigma$, we restrict its

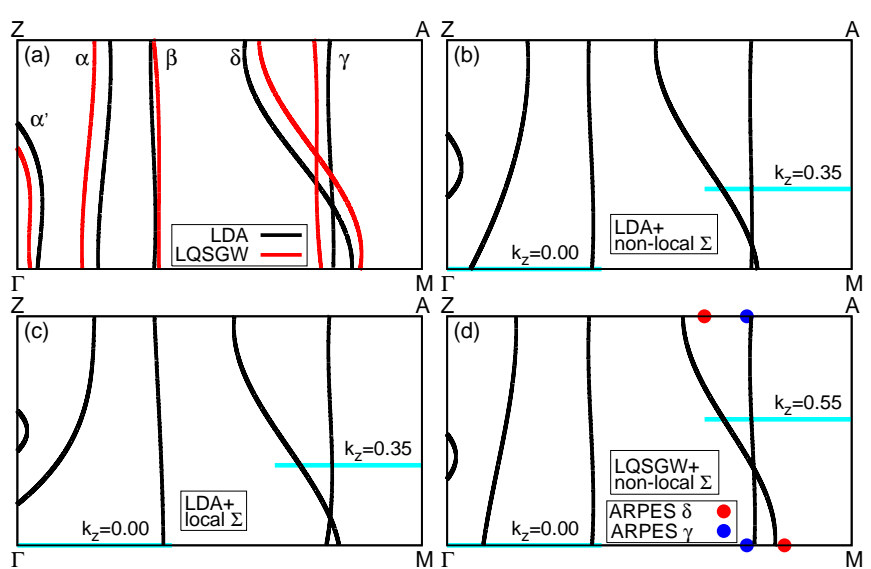

Figure 1. (a) Fermi surfaces of LDA (black) and LQSGW (red) in the $\Gamma-M-A-Z$ plane (b) Same as (a) for the LDA+non-local $\Sigma$ ansatz with $k_{z}=0.00$ for the hole bands fit and $k_{z}=0.35$ for the electron bands fit. (c) Same as (a) for the LDA+local $\Sigma$ ansatz with $k_{z}=0.00$ for the hole bands fit and $k_{z}=0.35$ for the electron bands fit. (d) Same as (a) for the LQSGW+non-local $\Sigma$ ansatz with $k_{z}=0.00$ for the hole bands fit and $k_{z}=0.55$ for the electron bands fit. Red and blue dots are the $\delta$ and $\gamma$ Fermi surfaces measured with ARPES in Ref. 44]. The ARPES data for fitting (b-d) is taken from Ref.[20, 45]. See Table \ for Fermi surface volumes.

functional form as follows. We assume that, in the local orbital basis, it is independent of the out-of-plane momentum $k_{z}$ and that the off-diagonal (inter-orbital) matrix elements are absorbed into the renormalization of the SOC 46 48]. Two different ansätze are made for the in-plane momentum dependence. (i) The self-energy components are simply assumed to be independent of momentum - we refer to this as the 'local $\Sigma$ ansatz'. (ii) The Brillouin zone is divided into two patches, centered around the $\Gamma$ - and $M$-points, respectively, as illustrated on Fig. 22 and a more flexible momentum dependence is allowed which is piecewise constant in each patch. We refer to this ansatz as the 'non-local $\Sigma$ ansatz' It corresponds to a two-site dynamical cluster approximation (DCA) which is a cluster extension of the DMFT [49]. These two ansätze thus read (see SM for details [43]):

$$
\begin{aligned}
\text { local } \Sigma \text { ansatz: } \Sigma_{m}(k, \omega) & =\Sigma_{m}(\omega) \\
\text { non-local } \Sigma \text { ansatz: } \Sigma_{m}(k, \omega) & =\Sigma_{m}(\Gamma, \omega) \text { if } k \in \Gamma \\
& =\Sigma_{m}(M, \omega) \text { if } k \in M(3)
\end{aligned}
$$

The components of the self-energy within the non-local $\Sigma$ ansatz are obtained by fitting the experimental hole bands at $\mathrm{K}=\Gamma$ and electron bands at $\mathrm{K}=M$ separately. We also note that this ansatz is physically motivated by the AFM wave-vector of spin fluctuations and corresponding Brillouin zone folding (Fig. 2(b)) 50]. We emphasize that these ansätze are made for the components of the self-energy expressed in the basis of local orbitals. The transformation to the quasiparticle (band) basis is momentum dependent and leads to significant 
(a) Brillouin zone and 2D Patch

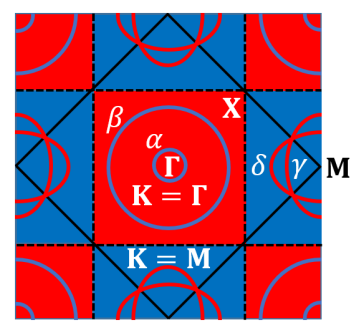

(b) 2D unit cell and AFM correlation

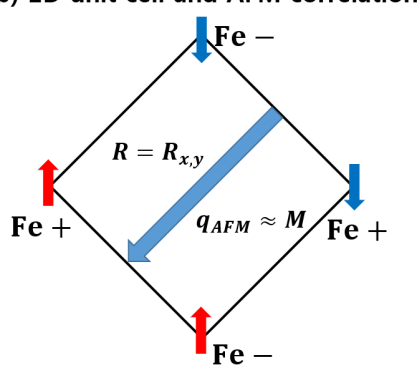

Figure 2. (a) Patching of the Brillouin zone for the nonlocal $\Sigma$ ansatz of LiFeAs. The solid line delimits the principal Brillouin zone (two irons in a unit cell), and the dashed lines indicate the patching used in the non-local $\Sigma$ ansatz. The patch centered on $\mathrm{K}=\Gamma$ (resp. $\mathrm{K}=M$ ) is colored in red (resp. blue). Schematic Fermi surfaces are represented by colored solid lines. Hole pockets are in blue, $\alpha$ (inner) and $\beta$ (outer). Electron pockets are in red, $\delta$ (outer) and $\gamma$ (inner). (b) Two-dimensional unit cell and the momentum $q_{A F M} \approx M$ associated with AFM correlations [50]. The AF-correlated Fe moments are schematized by the blue and red arrows, with $\mathrm{Fe}+$ and Fe- denoting the two Fe atoms in the unit cell.

momentum dependence of the self-energy in that basis even if a DMFT ansatz is made (see also Ref. [26]).

The assignment of $k_{z}$ from ARPES has uncertainties [51]. In our case, experiment constraints $k_{z}$ around the electron pockets to be in the interval $[0.3,0.7]$ while for the hole pockets, there is little uncertainty that the data arise from $k_{z}=0$ [45], (See $\operatorname{SM}[43]$ ). For the electron pockets, we considered two different ways to infer $k_{z}$. (i) The first is to require that the Fermi surface volume satisfies Luttinger's theorem, as obtained by a full Brillouin zone integration and assuming that the self-energy does not depend on $k_{z}$. As it turns out, for electron pockets, a unique value of $k_{z} \simeq 0.55$ satisfies this constraint for both ansätze. (ii) The second one determines $k_{z}$ by requesting that the resulting self energy is as local as possible. This leads to $k_{z}=0.35$ for the LDA+non-local $\Sigma$ ansatz and $k_{z}=0.55$ for the LQSGW+non-local $\Sigma$ ansatz (see $\mathrm{SM}[43])$. Note that in that case, Luttinger's theorem is violated within the LDA+non-local $\Sigma$ ansatz, while the value $k_{z}=0.55$ ensures both Luttinger's theorem and maximal locality when using the LQSGW reference.

Results. Our main results are summarized in Figs. 1)(bd), 3 and Tables I and II. The full frequency dependence of the self-energies extracted from the procedure described above is displayed on Fig.S5 in the supplemental material (SM) 43]. All results were obtained using the ARPES data of Ref 20, 45], also displayed on Fig. 3 [52]. The low-energy behaviour of the fitted self-energies is characterized by the zero-frequency (static) values $\Sigma_{m}(0)$, as well as the quasiparticle weights $Z_{m}=\left[1-\left.\frac{\partial \Sigma_{m}}{\partial \omega}\right|_{\omega=0}\right]^{-1}$, displayed in Table 【 Comparing the values obtained within the non-local $\Sigma$ ansatz for the $\Gamma$ - and $M$ - BZ patches, we see that, when starting

Table II. Zero frequency self-energy $\left(\Sigma_{m}(\mathrm{~K}, 0)\right)$ and quasiparticle residue $\left(\mathrm{Z}_{m}(\mathrm{~K})\right)$ extracted from ARPES data of LiFeAs 20, 45], with the LDA+non-local $\Sigma$ ansatz, the LQSGW+non-local $\Sigma$ ansatz, and the LDA+local $\Sigma$ ansatz. We use $k_{z}=0.00$ for $\mathrm{K}=\Gamma$ (hole sheets) for both the LDA and the LQSGW references, $k_{z}=0.35$ for $\mathrm{K}=M$ (electron sheets) for the LDA reference, and $k_{z}=0.55$ for $\mathrm{K}=M$ (electron sheets), for the LQSGW reference. Error bars (total) are computed from the peak width of both in plane $k$ and out of plane $k_{z}$. (See SM for the details on the definition of the error bars [43].)

\begin{tabular}{|c|c|c|c|c|}
\hline \multicolumn{5}{|c|}{ LDA+non-local $\Sigma$ ansatz } \\
\hline & $\Sigma_{m}(\Gamma, 0)(\mathrm{eV})$ & $\mathrm{Z}_{m}(\Gamma)$ & $\Sigma_{m}(M, 0)(\mathrm{eV})$ & $\mathrm{Z}_{m}(M)$ \\
\hline$x y$ & $0.029 \pm 0.025$ & $0.15 \pm 0.01$ & $-0.130 \pm 0.062$ & $0.12 \pm 0.01$ \\
\hline$x z / y z$ & $-0.083 \pm 0.040$ & $0.25 \pm 0.13$ & $-0.113 \pm 0.026$ & $0.16 \pm 0.03$ \\
\hline \multicolumn{5}{|c|}{$\stackrel{1}{L D A+\text { local } \Sigma \text { ansatz }}$} \\
\hline & \multicolumn{2}{|c|}{$\Sigma_{m}(0)(\mathrm{eV})$} & \multicolumn{2}{|l|}{$\mathrm{Z}_{m}$} \\
\hline$x y$ & \multirow{2}{*}{\multicolumn{2}{|c|}{0.023}} & \multicolumn{2}{|l|}{0.14} \\
\hline$x z / y z$ & & -0.112 & \multicolumn{2}{|l|}{0.17} \\
\hline \multicolumn{5}{|c|}{ LQSGW+non-local $\Sigma$ ansatz } \\
\hline & $\Sigma_{m}(\Gamma, 0)(\mathrm{eV})$ & $\mathrm{Z}_{m}(\Gamma)$ & $\Sigma_{m}(M, 0)(\mathrm{eV})$ & $\mathrm{Z}_{m}(M)$ \\
\hline$x y$ & $0.002 \pm 0.014$ & $0.21 \pm 0.01$ & $0.044 \pm 0.036$ & $0.18 \pm 0.01$ \\
\hline$x z / y z$ & $-0.027 \pm 0.003$ & $0.38 \pm 0.01$ & $-0.051 \pm 0.114$ & $0.30 \pm 0.04$ \\
\hline
\end{tabular}

from LDA, the static components of the self-energy are spatially local to a good approximation for the $x z / y z$ orbitals, while a higher degree of momentum-dependence holds for the $x y$ orbital. The quasiparticle weight associated with the $x y$ orbital is found to be weakly momentum dependent, while stronger momentum dependence is found for the $x z / y z$ orbital. This strong momentum dependence of the dynamical self-energy of the $x z / y z$ orbitals has been discussed in Refs. [5, 6, 19, 25] in relation to the strong coupling of the quasiparticles of the $x z / y z$ driven $\alpha$ and $\alpha^{\prime}$ hole-like FS sheets to the existing AFM correlation in LiFeAs [50]. Indeed, these FS sheets are close to the AFM zone boundary. The values of the quasiparticle weights obtained here, $(0.15(\Gamma) 0.12$ $(M)$ for $x y$ and $0.25(\Gamma) 0.16(M)$ for $x z / y z)$, are smaller than that of the computed LDA+DMFT values reported in Refs. [14, 16, 20] $\left(\mathrm{Z}_{x y}=0.26\right.$ and $\left.\mathrm{Z}_{x z / y z}=0.34\right)$. They are however close to the values $(0.17-0.19)$ reported by de Haas-van Alphen experiments 53.

Table II also displays the results obtained by using a local ansatz for the self-energies. As seen there, the values of the quasiparticle weights are intermediate between the values at the $\Gamma$ - and $M$-points obtained within the non-local ansatz.

Fig. 1 (b,c) displays how the FS is modified by selfenergy effects when using LDA as a starting point. Table Ireports the corresponding volume of each FS sheet. We see that both the local and non-local ansatz lead to a violation of the Luttinger theorem, when the value $k_{z}=0.35$ is used for the fitting of electron bands. This is mostly due to the large volume obtained for the $\delta$-sheet, which crosses the $\gamma$-sheet at a low value of $k_{z} \approx 0.05$ 
leading to a too large electron-like contribution.

We now turn to the results of the self-energy obtained by using LQSGW for the reference Hamiltonian, using $k_{z}=0.55$ in this case when fitting the electron bands around $M$. The results in Table clearly show that the fitted values of both $\Sigma_{m}(0)$ and $Z_{m}$ are quite momentum independent (spatially local) within the determined error bars. Some slight momentum dependence of $Z_{x z / y z}$ is found however ( $\sim 0.38$ at $\Gamma$-point vs $\sim 0.30$ at $M$-point $)$, close to the limit set by error bars. (See Fig.S5 of the SM 43. for the full frequency dependence of the extracted self-energies) Furthermore the Luttinger theorem is now well obeyed (Table I). This is due in particular to the much smaller inflation of the volume of the $\gamma$ and $\delta$ sheets by self-energy effects, in comparison to the LDA starting point. Correspondingly, the crossing point between the $\gamma$ and $\delta$ sheets occurs at a larger value of $k_{z}$ (Fig. 1(d)).

Comparing to available experimental data, we see that the LQSGW reference combined with a quasi-local selfenergy provides: (i) a good description of the $k_{z}$ dependent hole bands $\left(\alpha^{\prime}, \alpha\right.$, and $\beta$ ) dispersions in comparison to the ARPES data of Refs [20, 54] (see SM [43] for comparison), (ii) a good description of the $k_{z}$ dependent $\gamma$ FS in ARPES of Refs 44, 55], (iii) and a qualitative description of the $k_{z}$ dependent $\delta \mathrm{FS}$ in ARPES with correct $k_{z}$ for the crossing of the $\delta$ and $\gamma$ FSs and somewhat larger curvature of the $\delta$ FS near the momentum of A of Ref. 44, 45] Fig[1(d) implies that for electron bands, the overall amplitude of $k_{z}$ dependent variation of the $\delta$ and $\gamma$ FSs in the LQSGW+ fit is consistent with the ARPES data of Ref. 44].

We compare in Fig 3 (a-c) the experimental ARPES intensity to the fitted hole bands of LiFeAs using the different starting points $H(k)$ and ansatz for $\Sigma$. For the $x y$ dominant $\beta$ band, all schemes compare well with ARPES. In contrast, we observe some differences between the different ansätze (comparable to error bars) for the position of the top of the $\alpha$ band with dominant $x z / y z$ character. The LDA+local $\Sigma$ ansatz leads to a lower energy than the LDA+non-local $\Sigma$ ansatz and the LQSGW+nonlocal $\Sigma$ ansatz. The splitting of the states with $x z / y z$ character at the $\Gamma$ point is controlled by the SOC and given by $\lambda Z_{x z / y z}$ [46]. Its experimental value is $9.5-11.4$ $\mathrm{meV}[56,57]$. The values of $\lambda$ in LDA and LQSGW are $50 \mathrm{meV}$ and $25 \mathrm{meV}$ respectively, which when multiplied by the extracted $Z$ 's from Table II indeed leads to values close to $10 \mathrm{meV}$ in both cases (see SM for details on the effect of SOC in LiFeAs [43]).

We now turn to the electron bands in Fig 3 (d-i). Along the $\Gamma-M$ direction, the $\gamma$ band has almost pure $x y$ character, and is seen in $\sigma$ polarized ARPES. This $\gamma$ band is well described by both the non-local ansatz and the local ansatz within both references (LDA and LQSGW), see panels Fig 3 (g,h, and i). For the $x z / y z$ dominant $\delta$ band, the LDA+non-local $\Sigma$ ansatz and the LQSGW+nonlocal $\Sigma$ ansatz yield quasiparticle spectra which are con-
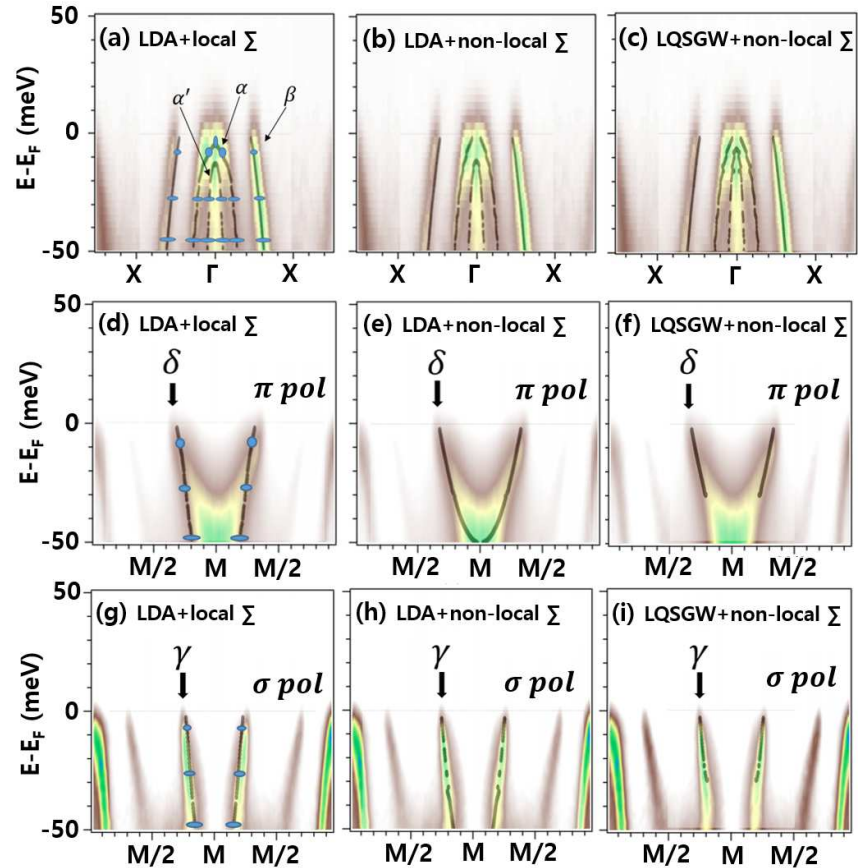

Figure 3. Comparison between the ARPES data of Ref. 20, 45] for LiFeAs (color intensity map) with different polarizations $(\pi$ and $\sigma$ ) and the quasiparticle dispersions obtained with the different ansätze discussed in the text. The results of LDA+local $\Sigma$, LDA+non-local $\Sigma$, and LQSGW+non-local $\Sigma$ ansätze are shown in panels (a, d and g), (b, e and h), and (c, f and i), respectively. The hole pocket data are taken at $k_{z}=0.00$ and the electron pockets data are taken at a value of $k_{z}$ within the range of $[0.3,0.7][20,45]$. The blue colored ellipses are theoretical error estimates arising from the width of the MDC peak and the uncertainty in $k_{z}$. For the electron bands, $k_{z}=0.55$ has been used in the fit using LQSGW as a reference, while $k_{z}=0.35$ when using LDA as a reference (see main text).

sistent with ARPES within error bars as shown in Fig 3 (e, and $\mathrm{f}$ ). However, differences between the fits are seen for the $x z / y z$ driven $\delta$ band with the LDA + local $\Sigma$ ansatz having a steeper dispersion and a lower bottom than the non-local $\Sigma$ ansatz, as seen in Fig 3(d, e, and f). Also, as noted in Table I and Fig [1(b,c), this fit violates the Luttinger's theorem.

In summary, our analysis demonstrates that an LQSGW reference 29, 36, 58] in combination with quite local self-energies provides a description of the quasiparticle dispersions of LiFeAs in good agreement with experiments. The strong dispersion along $k_{z}$ of the $\alpha$ and $\delta$ FS sheets, unique to the 111 compounds, is also well described, although the latter is slightly overestimated. Hence, correlation effects can be decomposed into nonlocal, frequency independent contributions captured by the LQSGW and dynamical frequency-dependent contributions that are spatially local to a good approximation. In contrast, when using LDA as a reference, the extracted self-energy is spatially non-local and, when taken to be 
$k_{z}$-independent, leads to an overestimation of the volume of the FS $\delta$-sheet and a corresponding violation of Luttinger theorem. We emphasize that, in contrast to theories attributing non-locality to AFM spin fluctuations, the non-locality in the LQSGW approach originates from the charge sector.

We finally turn to transport measurements, as reported in Ref. [59], and investigate whether our LQSGW+local $\Sigma$ analysis is consistent with those data. Using the occupancies of the different FS sheets obtained above, we use the experimental data for the resistivity and Hall effect to obtain the scattering rates associated with each orbital component, as a function of temperature, under the assumption that they are spatially local. The conclusion of this analysis (see details in SM [43]) is that the $x y$ orbital is found to have a larger scattering rate than the $x z / y z$ one, and that it undergoes a clear crossover at $T \sim 150 \mathrm{~K}$ between a high- $T$ incoherent regime to a low- $T$ coherent one. This is consistent with the LQSGW+local $\Sigma$ finding that the $x y$ orbital is the more correlated one. At low- $T$ both scattering rates are found to have a Fermi liquid $T^{2}$ behaviour. As a consistency check, we also obtain satisfactory agreement with the magnetoresistance data. Let us emphasize that, in contrast, studies emphasizing non-locality due to low-energy AFM fluctuations yield a non-Fermi liquid scattering rate of the $x z / y z$ orbital which is larger than that of $x y[\underline{5},[6]$.

Several authors have pointed at some discrepancies between experimental data and the predictions of LDA+DMFT, which is usually interpreted as a failure of the DMFT to take into account non-local effects [5, 6, 24, 25, 60]. Here, based on a direct analysis of ARPES experimental data, we presented a very different picture, consistent with the electronic structure+DMFT conceptual framework. We have shown that when we start from the LQSGW reference Hamiltonian, the low energy self-energy is spatially local, satisfies Luttinger's theorem, describes available experimental data well and therefore is an attractive platform to study how superconductivity emerges at lower temperatures. 9, 1113, 15, 18.

We acknowledge useful discussions with Andrea Damascelli and Ryan Day (who also kindly shared their unpublished ARPES data) as well as with Roser Valenti. This work was supported by the DOE CMS program (MK and GK). SC was supported by the U.S Department of Energy, Office of Science, Basic Energy Sciences as a part of the Computational Materials Science Program. For the LQSGW calculation, we used resources of the National Energy Research Scientific Computing Center (NERSC), a U.S. Department of Energy Office of Science User Facility operated under Contract No. DE-AC02-05CH11231. HM was supported by the Laboratory Directed Research and Development Program of Oak Ridge National Laboratory, managed by UT-Battelle, LLC, for the U.S. Department of Energy. AG acknowledges the support of the
European Research Council (ERC-319286-QMAC). The Flatiron Institute is a division of the Simons Foundation.

* garix.minjae.kim@gmail.com

[1] Y. Kamihara, H. Hiramatsu, M. Hirano, R. Kawamura, H. Yanagi, T. Kamiya, and H. Hosono, Journal of the American Chemical Society 128, 10012 (2006).

[2] I. Mazin, D. J. Singh, M. Johannes, and M.-H. Du, Physical Review Letters 101, 057003 (2008).

[3] K. Kuroki, S. Onari, R. Arita, H. Usui, Y. Tanaka, H. Kontani, and H. Aoki, Physical Review Letters 101, 087004 (2008).

[4] A. V. Chubukov, D. Efremov, and I. Eremin, Physical Review B 78, 134512 (2008).

[5] K. Zantout, S. Backes, and R. Valentí, Physical Review Letters 123, 256401 (2019).

[6] J. Fink, J. Nayak, E. Rienks, J. Bannies, S. Wurmehl, S. Aswartham, I. Morozov, R. Kappenberger, M. ElGhazali, L. Craco, et al., Physical Review B 99, 245156 (2019).

[7] P. Dai, Reviews of Modern Physics 87, 855 (2015).

[8] L. Fanfarillo, E. Cappelluti, C. Castellani, and L. Benfatto, Physical review letters 109, 096402 (2012).

[9] K. Umezawa, Y. Li, H. Miao, K. Nakayama, Z.-H. Liu, P. Richard, T. Sato, J. He, D.-M. Wang, G. Chen, et al., Physical Review Letters 108, 037002 (2012).

[10] S. Hoshino and P. Werner, Physical Review Letters 115, 247001 (2015).

[11] H. Miao, W. Brito, Z. Yin, R. Zhong, G. Gu, P. Johnson, M. Dean, S. Choi, G. Kotliar, W. Ku, et al., Physical Review B 98, 020502 (2018).

[12] T.-H. Lee, A. Chubukov, H. Miao, and G. Kotliar, Physical Review Letters 121, 187003 (2018).

[13] P. Coleman, Y. Komijani, and E. J. König, Physical Review Letters 125, 077001 (2020).

[14] Z. Yin, K. Haule, and G. Kotliar, Nature materials 10, 932 (2011).

[15] H. Miao, P. Richard, Y. Tanaka, K. Nakayama, T. Qian, K. Umezawa, T. Sato, Y.-M. Xu, Y. Shi, N. Xu, et al., Physical Review B 85, 094506 (2012).

[16] G. Lee, H. S. Ji, Y. Kim, C. Kim, K. Haule, G. Kotliar, B. Lee, S. Khim, K. H. Kim, K. S. Kim, et al., Physical Review Letters 109, 177001 (2012).

[17] P. Werner, M. Casula, T. Miyake, F. Aryasetiawan, A. J. Millis, and S. Biermann, Nature Physics 8, 331 (2012).

[18] Z. P. Yin, K. Haule, and G. Kotliar, Nature Physics 10, 845 (2014).

[19] H. Miao, T. Qian, X. Shi, P. Richard, T. Kim, M. Hoesch, L. Xing, X.-C. Wang, C.-Q. Jin, J.-P. Hu, et al., Nature communications 6, 1 (2015).

[20] H. Miao, Z. Yin, S. Wu, J. Li, J. Ma, B.-Q. Lv, X. Wang, T. Qian, P. Richard, L.-Y. Xing, et al., Physical Review B 94, 201109 (2016).

[21] P. Sémon, K. Haule, and G. Kotliar, Physical Review B 95, 195115 (2017).

[22] J. M. Tomczak, M. van Schilfgaarde, and G. Kotliar, Physical Review Letters 109, 237010 (2012).

[23] J. Ferber, K. Foyevtsova, R. Valentí, and H. O. Jeschke, Physical Review B 85, 094505 (2012).

[24] L. Ortenzi, E. Cappelluti, L. Benfatto, and L. Pietronero, 
Physical Review Letters 103, 046404 (2009).

[25] S. Bhattacharyya, K. Björnson, K. Zantout, D. Steffensen, L. Fanfarillo, A. Kreisel, R. Valentí, B. M. Andersen, and P. Hirschfeld, arXiv preprint arXiv:2003.01638 (2020).

[26] A. Tamai, M. Zingl, E. Rozbicki, E. Cappelli, S. Ricco, A. de la Torre, S. M. Walker, F. Bruno, P. King, W. Meevasana, et al., Physical Review X 9, 021048 (2019).

[27] J. H. Tapp, Z. Tang, B. Lv, K. Sasmal, B. Lorenz, P. C. Chu, and A. M. Guloy, Physical Review B 78, 060505 (2008).

[28] T. Mertz, K. Zantout, and R. Valentí, Physical Review B 98, 235105 (2018).

[29] J. M. Tomczak, Journal of Physics: Conference Series 592, 012055 (2015).

[30] A. L. Kutepov, V. S. Oudovenko, and G. Kotliar, Computer Physics Communications 219, 407 (2017).

[31] A. Kutepov, K. Haule, S. Y. Savrasov, and G. Kotliar, Physical Review B 85, 155129 (2012).

[32] A. Georges, G. Kotliar, W. Krauth, and M. J. Rozenberg, Review of Modern Physiscs 68, 13 (1996)

[33] G. Kotliar, S. Y. Savrasov, K. Haule, V. S. Oudovenko, O. Parcollet, and C. Marianetti, Reviews of Modern Physics 78, 865 (2006).

[34] M. Qazilbash, J. Hamlin, R. Baumbach, L. Zhang, D. J. Singh, M. Maple, and D. Basov, Nature Physics 5, 647 (2009).

[35] L. de' Medici, G. Giovannetti, and M. Capone, Physical Review Letters 112, 177001 (2014).

[36] S. Choi, P. Semon, B. Kang, A. Kutepov, and G. Kotliar, Computer Physics Communications 244, 277 (2019).

[37] P. Blaha, K. Schwarz, G. K. Madsen, D. Kvasnicka, and J. Luitz, An augmented plane wave+ local orbitals program for calculating crystal properties (2001).

[38] P. Blaha, K. Schwarz, F. Tran, R. Laskowski, G. K. Madsen, and L. D. Marks, The Journal of Chemical Physics 152, 074101 (2020).

[39] I. Souza, N. Marzari, and D. Vanderbilt, Physical Review B 65, 035109 (2001).

[40] N. Marzari and D. Vanderbilt, Physical review B 56, 12847 (1997).

[41] A. A. Mostofi, J. R. Yates, Y.-S. Lee, I. Souza, D. Vanderbilt, and N. Marzari, Computer physics communications 178, 685 (2008).

[42] J. Kuneš, R. Arita, P. Wissgott, A. Toschi, H. Ikeda, and K. Held, Computer Physics Communications 181, 1888 (2010).

[43] See Supplemental Material (SM) for (i) information on the construction of $H(k)$ with maximally localized Wannier function (MLWF) for DFT-LDA and LQSGW, (ii) details of the microscopic calculations include SOC, (iii) $k_{z}$ dependent electron band dispersions of $H(k)$, (iv) details of the method for the extraction of the self-energy including error bars, (v) comparison of the quality of the present LQSGW+non-local $\Sigma$ fit to the published ARPES data, showing that the fitting of the hole pockets leads to a good descriptions of the published data for $k_{z}=0.00$ [20] and for other values of $k_{z}$. [54], (vi) self- energy depending on the assigned $k_{z}$ value for electron pockets and corresponding Fermi surface volumes for the Luttinger's theorem, (vii) frequency dependency of the dynamical self-energy, and (viii) analysis of the transport data of Ref. [59] and discussions of the extracted scattering rate from the transport data.

[44] V. Brouet, D. LeBoeuf, P.-H. Lin, J. Mansart, A. TalebIbrahimi, P. Le Fèvre, F. Bertran, A. Forget, and D. Colson, Physical Review B 93, 085137 (2016).

[45] Hu Miao, unpublished.

[46] M. Kim, J. Mravlje, M. Ferrero, O. Parcollet, and A. Georges, Physical Review Letters 120, 126401 (2018).

[47] N.-O. Linden, M. Zingl, C. Hubig, O. Parcollet, and U. Schollwöck, Physical Review B 101, 041101 (2020).

[48] A. Horvat, J. Mravlje, et al., Physical Review B 96, $085122(2017)$.

[49] T. Maier, M. Jarrell, T. Pruschke, and M. H. Hettler, Reviews of Modern Physics 77, 1027 (2005).

[50] N. Qureshi, P. Steffens, Y. Drees, A. Komarek, D. Lamago, Y. Sidis, L. Harnagea, H.-J. Grafe, S. Wurmehl, B. Büchner, et al., Physical Review Letters 108, 117001 (2012).

[51] A. Damascelli, Physica Scripta 2004, 61 (2004).

[52] The ARPES data has been measured at $20 \mathrm{~K}$ which is slightly above the superconducting transition temperature $(18 \mathrm{~K})$ of LiFeAs. 27.

[53] C. Putzke, A. Coldea, I. Guillamón, D. Vignolles, A. McCollam, D. LeBoeuf, M. Watson, I. Mazin, S. Kasahara, T. Terashima, et al., Physical Review Letters 108, 047002 (2012).

[54] Z. Wang, P. Zhang, G. Xu, L. Zeng, H. Miao, X. Xu, T. Qian, H. Weng, P. Richard, A. Fedorov, et al., Physical Review B 92, 115119 (2015).

[55] T. Hajiri, T. Ito, R. Niwa, M. Matsunami, B. Min, Y. Kwon, and S. Kimura, Physical Review B 85, 094509 (2012).

[56] S. Borisenko, D. Evtushinsky, Z.-H. Liu, I. Morozov, R. Kappenberger, S. Wurmehl, B. Büchner, A. Yaresko, T. Kim, M. Hoesch, et al., Nature Physics 12, 311 (2016).

[57] R. Day, G. Levy, M. Michiardi, B. Zwartsenberg, M. Zonno, F. Ji, E. Razzoli, F. Boschini, S. Chi, R. Liang, et al., Physical Review Letters 121, 076401 (2018).

[58] S. Choi, A. Kutepov, K. Haule, M. van Schilfgaarde, and G. Kotliar, npj Quantum Materials 1, 16001 (2016).

[59] F. Rullier-Albenque, D. Colson, A. Forget, and H. Alloul, Physical Review Letters 109, 187005 (2012).

[60] S. Borisenko, V. Zabolotnyy, D. Evtushinsky, T. Kim, I. Morozov, A. Yaresko, A. Kordyuk, G. Behr, A. Vasiliev, R. Follath, et al., Physical Review Letters 105, 067002 (2010).

[61] J. Kuneš, R. Arita, P. Wissgott, A. Toschi, H. Ikeda, and K. Held, Computer Physics Communications 181, 1888 (2010).

[62] M. J. Pitcher, D. R. Parker, P. Adamson, S. J. Herkelrath, A. T. Boothroyd, R. M. Ibberson, M. Brunelli, and S. J. Clarke, Chemical Communications , 5918 (2008).

[63] A. Kutepov, K. Haule, S. Y. Savrasov, and G. Kotliar, 85, $155129(2012)$

[64] A. Kutepov, V. Oudovenko, and G. Kotliar, 219, 407 (2017) 


\title{
Supplemental Material: \\ On the Spatial Locality of Electronic Correlations in LiFeAs
}

\author{
Minjae Kim ${ }^{1,2}$, Hu Miao $^{3}$, Sangkook $\mathrm{Choi}^{4}$, Manuel Zingl ${ }^{5}$, Antoine Georges ${ }^{6,5,7,8}$, and Gabriel Kotliar ${ }^{1,4}$ \\ ${ }^{1}$ Department of Physics and Astronomy, Rutgers University, Piscataway, New Jersey 08854, USA \\ ${ }^{2}$ Department of Chemistry, Pohang University of Science and Technology (POSTECH), Pohang 37673, Korea \\ ${ }^{3}$ Materials Science and Technology Division, Oak Ridge National Laboratory, Oak Ridge, Tennessee 37831, USA \\ ${ }^{4}$ Condensed Matter Physics and Materials Science Department, Brookhaven National Laboratory, Upton, New York 11973, \\ USA \\ ${ }^{5}$ Center for Computational Quantum Physics, Flatiron Institute, 162 5th Avenue, New York, NY 10010, USA \\ ${ }^{6}$ Collège de France, 11 place Marcelin Berthelot, 75005 Paris, France \\ ${ }^{7}$ Centre de Physique Théorique, École Polytechnique, CNRS, Université Paris-Saclay, 91128 Palaiseau, France \\ ${ }^{8}$ Department of Quantum Matter Physics, University of Geneva, 24 Quai Ernest-Ansermet, 1211 Geneva 4, Switzerland
}

\section{CONSTRUCTION OF THE REFERENCE HAMILTONIAN OF $H^{L D A}$}

In this section, we describe details of the construction of the reference Hamiltonian in the LDA, $H^{L D A}$. We construct maximally localized Wannier function (MLWF) of LiFeAs for the $p-d$ model approach $(\mathrm{Fe}(d)$ and $\operatorname{As}(p))$ using the Wannier90 and the Wien2Wannier packages. [S41, S61] We use the energy window of the interval of -6.0 $\mathrm{eV}$ to $3.0 \mathrm{eV}$ for $\mathrm{Fe}(d)$ and $\mathrm{As}(p)$ bands. We used the experimental crystal structure of LiFeAs of the Ref. [S62]. For the convergence of the charge density in the LDA, we used a $k$-mesh of 10000 , and checked that the charge density and the total energy are converged with criterions of $5 \times 10^{-4}$ (electrons/formula unit) and 0.7 (meV/formula unit), respectively. For the construction of MLWF, we used a $k$-mesh of $11 \times 11 \times 7$. The local axis for the MLWF of Fe $(d)$ is chosen such that (i) $z$ along $c$ of the unit cell, and (ii) $x$ and $y$ axes toward nearest neighboring Fe atoms. For the $p-d$ model, the spread function of the MLWF is converged as (i) $0.985,1.073,1.111$, and $1.333\left(\AA^{2}\right)$ for $z^{2}, x^{2}-y^{2}$, $x y$, and $x z / y z$ orbitals of Fe, and (ii) 3.197, and $3.301\left(\AA^{2}\right)$ for $p_{z}$, and $p_{x, y}$ orbitals of As.

Fig $\mathbf{S 1}$ presents Fermi surfaces and low energy band structures of $p-d$ model $+\lambda_{S O C}\left(\mathrm{LDA}+\lambda_{S O C}\right)$ in comparison with LDA plus SOC (LDA+SOC) with $\lambda_{S O C}=50 \mathrm{meV}$. This data implies that the electronic structure of the $p-d$ model $+\lambda_{S O C}\left(\mathrm{LDA}+\lambda_{S O C}\right)$ and $\mathrm{LDA}+\mathrm{SOC}$ are consistent. The factor that the local SOC is implemented only in the $\mathrm{Fe}(d)$ orbital in the $p-d$ model $+\lambda_{S O C}\left(\mathrm{LDA}+\lambda_{S O C}\right)$ proves that the SOC in the LDA+SOC is fully Fe $(d)$ orbital driven.

(a) Fermi surface, $p-d$ model $+\lambda_{\text {soc }}$

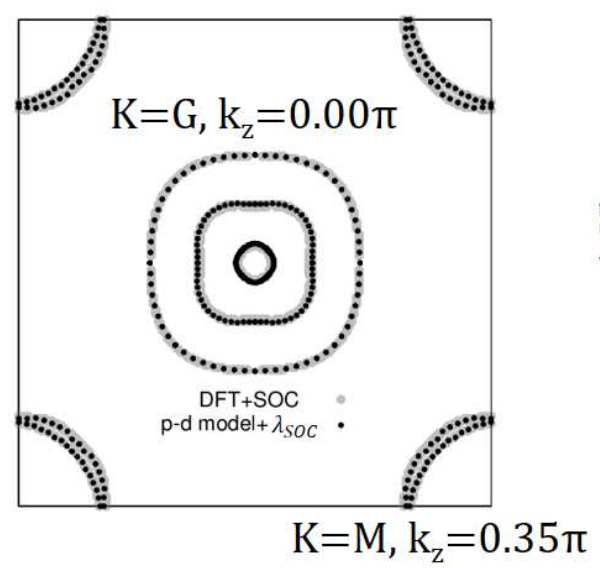

(b) Band structure, $p-d$ model $+\lambda_{\text {soc }}$

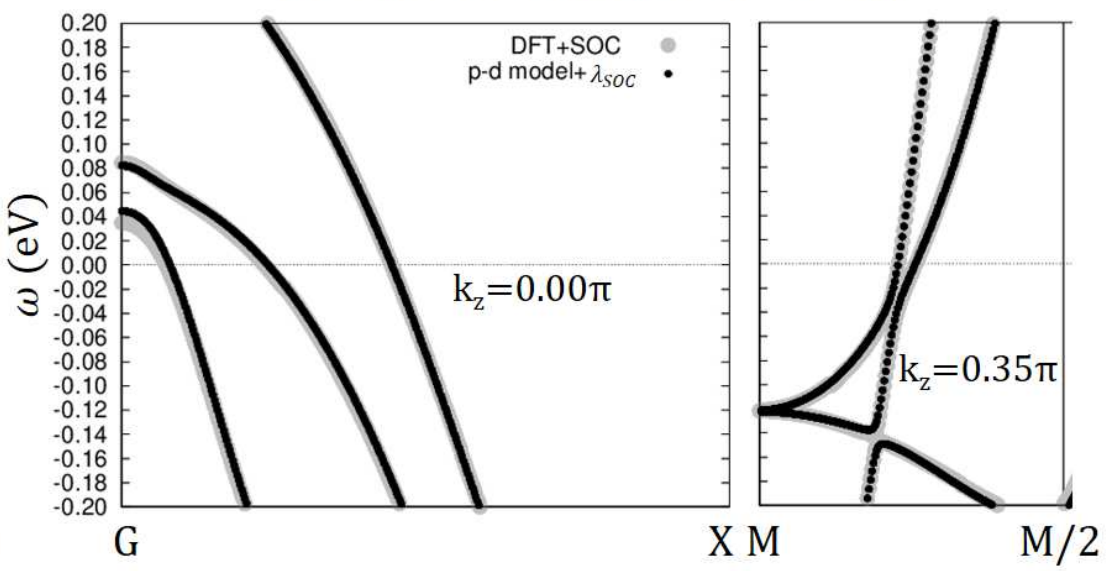

Figure S1. (a) Comparison of Fermi surfaces of the LDA+SOC and the p-d model $+\lambda_{S O C}\left(\mathrm{LDA}+\lambda_{S O C}\right)$. (b) Comparison of band dispersions of the LDA $+\mathrm{SOC}$ and the p-d model $+\lambda_{S O C}\left(\mathrm{LDA}+\lambda_{S O C}\right)$. The effective $\mathrm{SOC}$ of $\mathrm{Fe}(d)$ is $\lambda_{S O C}=50 \mathrm{meV}$. Here we take $k_{z}=0.00$ for hole bands, and $k_{z}=0.35$ for electron bands. 


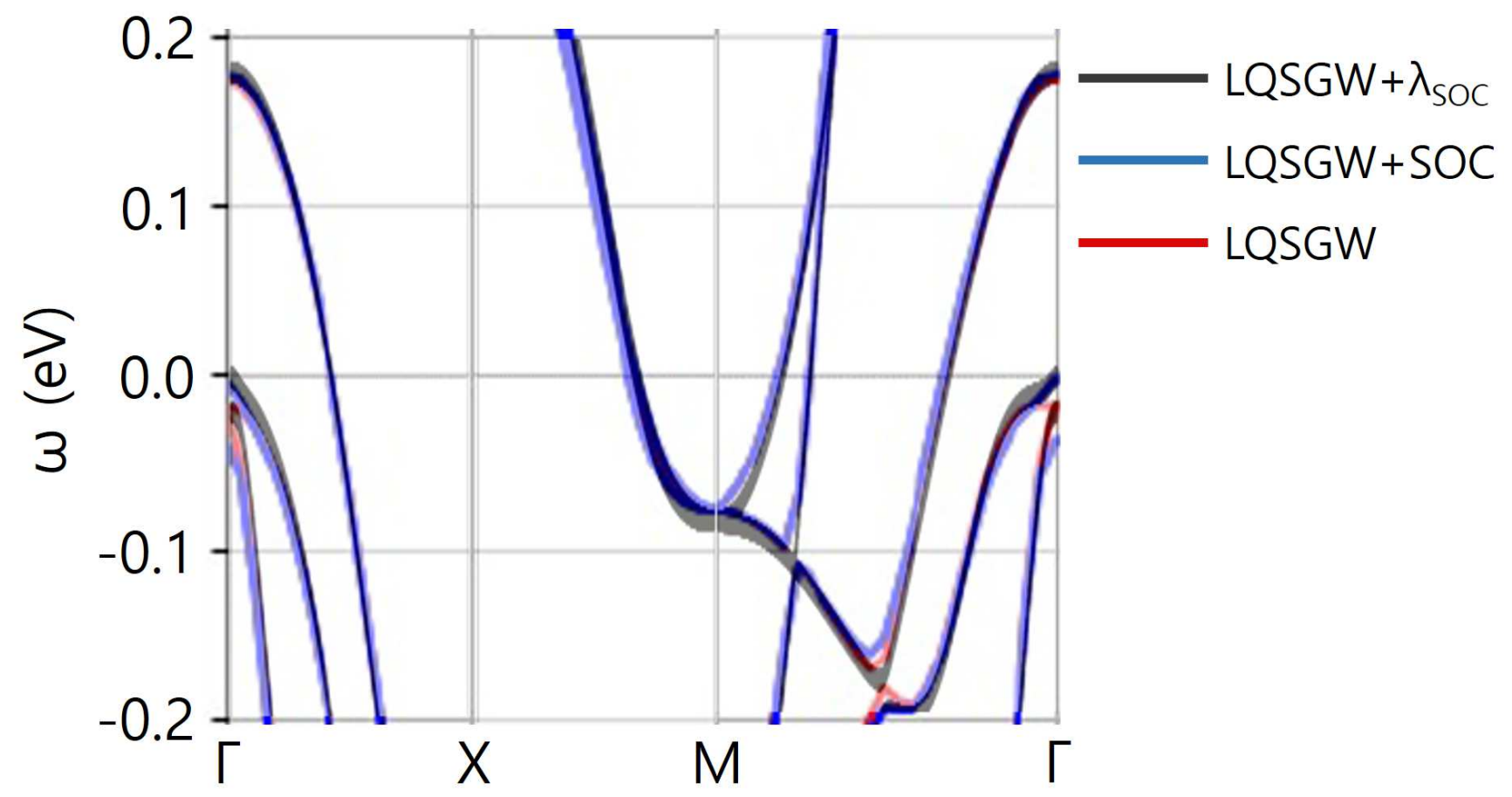

Figure S2. Comparison of band dispersions of the LQSGW $+\lambda_{S O C}\left(\lambda_{S O C}=25 \mathrm{meV}\right)$, LQSGW+SOC, and LQSGW. Here, the chemical potential is $-30 \mathrm{meV}$ for the Luttinger's theorem.

\section{CONSTRUCTION OF THE REFERENCE HAMILTONIAN OF $H^{L Q S G W}$}

In this section, we describe details of the construction of the reference Hamiltonian in the LQSGW, $H^{L Q S G W}$. LQSGW calculation is performed by using FlapwMBPT package [S63, S64], which is based on full-potential linearized augmented plane wave plus local orbital method. For the crystal structure, experimental lattice constants and atomic positions [S27] are used. The Muffin-tin (MT) radius $(R)$ is chosen in the following way: 1.74 for Li, 2.27 for Fe, and 2.16 for As in Bohr radius. Wave functions are expanded by spherical harmonics with 1 up to 3 for $\mathrm{Li}, 4$ for $\mathrm{Fe}$, and 4 for As in the MT spheres. In the interstitial region (IS), it is expanded by plane waves with the cutoff $\left(K_{c u t}\right)$ of $R_{F e} \times K_{c u t}=4$.4. Product basis set is expanded by spherical harmonics with 1 up to 4 in the MT spheres and by planewaves with the cutoff $\left(G_{c u t}\right)$ of $R_{F e} \times G_{c u t}=7.4$ in IS region. All the unoccupied states are taken into account for both polarizability and self-energy calculation. The Brillioun zone is sampled in $6 \times 6 \times 4$ grid.

By using ComWann modules in ComDMFT package [S36] utilizing Wannier90 package [S41], 42 wannier functions are constructed: Li-p, Fe-s, Fe-p, Fe-d, As-s, As-p, and As-d orbitals. The frozen energy window is set between $-9 \mathrm{eV}$ to $7 \mathrm{eV}$ and the disentanglement energy window is between $-9 \mathrm{eV}$ to $49 \mathrm{eV}$. Initial trial orbitals are constructed by using Muffin-tin orbitals with well-defined angular momentum characters. The local axis for the MLWF of Fe $(d)$ is chosen such that (i) $z$ along $c$ of the unit cell, and (ii) $x$ and $y$ axes toward nearest neighboring Fe atoms. The spread function of the MLWF is converged as (i) $0.497,0.493,0.524$, and $0.522\left(\AA^{2}\right)$ for $z^{2}, x^{2}-y^{2}, x y$, and $x z / y z$ orbitals of Fe, and (ii) 1.499 , and $1.724\left(\AA^{2}\right)$ for $p_{z}$, and $p_{x, y}$ orbitals of As.

Fig $\$ 2$ presents low energy band structures of the LQSGW $+\lambda_{S O C}$ with local SOC for Fe(d) (with $\lambda_{S O C}=25 \mathrm{meV}$ ) in comparison with the LQSGW plus SOC (LQSGW+SOC). The overall consistency in between the band structure of the LQSGW+SOC and the LQSGW $+\lambda_{S O C}$ implies that the local SOC in $\mathrm{Fe}(d)$ is a good approximation. The splitting of $\alpha$ and $\alpha$, bands at $\Gamma$ is $35 \mathrm{meV}$ for the LQSGW+SOC and $25 \mathrm{meV}$ for the LQSGW $+\lambda_{S O C}$, respectively (See Table S1). In Fig S2 it is shown that dispersions of $\alpha, \beta, \delta$, and $\gamma$ bands are agreement in between the LQSGW+SOC and the LQSGW $+\lambda_{S O C}$. These $\alpha, \beta, \delta$, and $\gamma$ bands are taken for the extraction of the self-energy. 

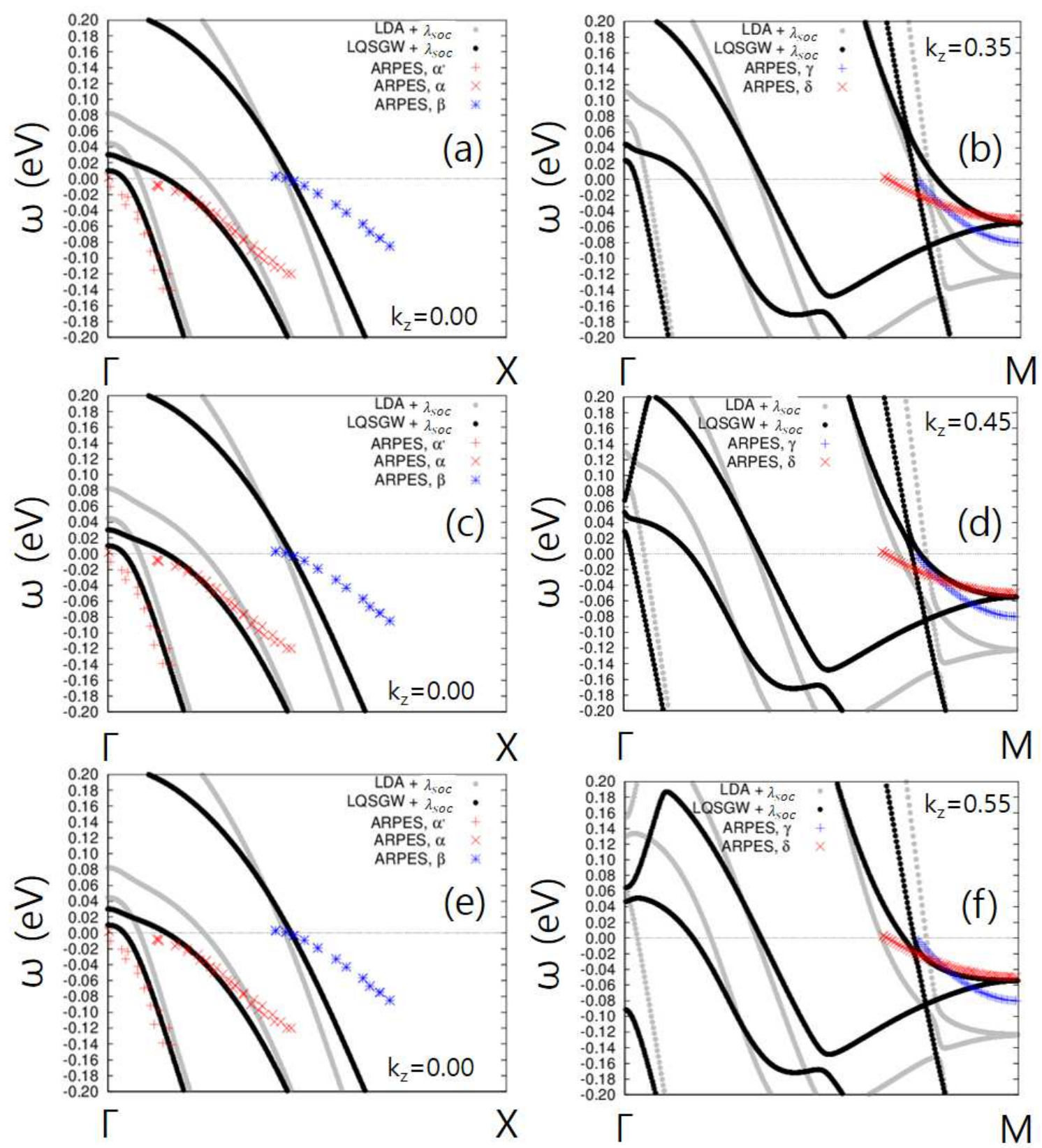

Figure S3. (a) and (b) comparison of the ARPES data to the (i) LDA reference $\left(\lambda_{S O C}=50 \mathrm{meV}\right)$ and the (ii) LQSGW reference $\left(\lambda_{S O C}=25 \mathrm{meV}\right)$ with $k_{z}=0.00$ for hole pockets $\left(\alpha, \alpha^{\prime}\right.$, and $\left.\beta\right)$ and $k_{z}=0.35$ for electron pockets $(\delta$ and $\gamma)$. (c) and (d) same as (a) and (b) with $k_{z}=0.00$ for hole pockets $\left(\alpha, \alpha^{\prime}\right.$, and $\beta$ ) and $k_{z}=0.45$ for electron pockets $(\delta$ and $\gamma)$. (e) and (f) same as (a) and (b) with $k_{z}=0.00$ for hole pockets $\left(\alpha, \alpha^{\prime}\right.$, and $\left.\beta\right)$ and $k_{z}=0.55$ for electron pockets $(\delta$ and $\gamma)$.

\section{ARPES VERSUS BAND STRUCTURES $H(k)$ OF LDA AND LQSGW}

In this section, we describe comparisons of bands in between ARPES and $H(k)$ of LDA and LQSGW to provide background information for the extraction of self-energy.

Fig $[$ 3 presents comparison of ARPES ( $[20]$, see the main text), to the band structures of $H(k)$ of LDA and LQSGW. The case of $k_{z}=0.00$ is shown for hole pockets for the extraction of self-energy. The case of $k_{z}=0.35-0.55$ is shown for the electron pockets to show the effect of the choice of $k_{z}$ for the extraction of the self-energy.

$L D A$ reference-For hole pockets with $k_{z}=0.00$, following factors are shown in Fig $[$ S3 (a,c, and e). (i) There is an agreement of the size of the $x y$ driven $\beta$ Fermi surface of $H^{L D A}(k)$ to the ARPES. (ii) There is a significant shrinking of the size of $x z / y z$ driven $\alpha$ and $\alpha^{\prime}$ Fermi surfaces in the ARPES with respect to those Fermi surfaces 
from $H^{L D A}(k)$. (iii) There are renormalizations of bands in the ARPES from the $H^{L D A}(k)$ for $\alpha, \alpha^{\prime}$, and $\beta$ bands. For electron pockets with $k_{z}=0.35$, following factors are shown in Fig S3(b). (i) There is a shrinking of the $x z / y z$ driven $\delta$ band in the ARPES with respect to the band from $H^{L D A}(k)$, with a similar amount with respect to the case of $x z / y z$ driven $\alpha$ and $\alpha^{\prime}$. (ii) There is a small shrinking of the $x y$ driven $\gamma$ band in the ARPES with respect to the band from $H^{L D A}(k)$. (iii) There are renormalizations of bands in the ARPES from the $H^{L D A}(k)$ for $\delta$ and $\gamma$ bands. For electron pockets with $k_{z}=0.55$, the $x y$ driven $\gamma$ band of $H^{L D A}(k)$ have a similar feature with respect to the case with $k_{z}=0.35$, as shown in Fig S3 (b and f). For the electron band $\delta$ with $k_{z}=0.55$, for the size of the Fermi momentum, there is an agreement in between the ARPES and the $H^{L D A}(k)$ as shown in Fig $\mathbf{S 3}$ (f). For $k_{z}=0.55$ for electron pockets, there are also renormalizations of bands in ARPES with respect to that in the $H^{L D A}(k)$ as shown in Fig 3 (f).

$L Q S G W$ reference-For hole pockets with $k_{z}=0.00$, following factors are shown in Fig 3 (a,c, and e). (i) There is an agreement of the size of the $x y$ driven $\beta$ Fermi surface of $H^{L Q S G W}(k)$ with the ARPES. (ii) There is a small shrinking of the size of $x z / y z$ driven $\alpha$ and $\alpha^{\prime}$ Fermi surfaces in the ARPES with respect to those Fermi surfaces from $H^{L Q S G W}(k)$. (iii) There are renormalizations of bands in the ARPES from the $H^{L Q S G W}(k)$ for $\alpha, \alpha^{\prime}$, and $\beta$ bands. For electron pockets with $k_{z}=0.55$, following factors are shown in Fig S3(f). (i) There is a small shrinking of the $x z / y z$ driven $\delta$ band in the ARPES with respect to the band from $H^{L Q S G W}(k)$, with a similar amount with respect to the case of $x z / y z$ driven $\alpha$ and $\alpha^{\prime}$. (ii) There is an agreement of the Fermi momentum of the $x y$ driven $\gamma$ band in the ARPES with respect to the band from $H^{L Q S G W}(k)$. (iii) There are renormalizations of bands in the ARPES from the $H^{L Q S G W}(k)$ for $\delta$ and $\gamma$ bands. For electron pockets with $k_{z}=0.35$, following factors are shown in Fig S3(b). (i) There is a significant shrinking of the $x z / y z$ driven $\delta$ band in the ARPES with respect to the band from $H^{L Q S G W}(k)$, with a much larger amount with respect to the case of $x z / y z$ driven $\alpha$ and $\alpha^{\prime}$. (ii) There is an agreement of the Fermi momentum of the $x y$ driven $\gamma$ band in the ARPES with respect to the band from $H^{L Q S G W}(k)$. (iii) There are renormalizations of bands in the ARPES from the $H^{L Q S G W}(k)$ for $\delta$ and $\gamma$ bands.

\section{EXTRACTION OF THE SELF-ENERGY}

In this section, we describe the procedure for the extraction of the self-energy of LiFeAs. For $H(k)$, we assume the reference Hamiltonian of $\mathrm{LDA}+\lambda_{S O C}\left(\lambda_{S O C}=50 \mathrm{meV}\right)$ and LQSGW $+\lambda_{S O C}\left(\lambda_{S O C}=25 \mathrm{meV}\right)$ as described in sections I and II.

In order to extract the self-energy (real part) from the ARPES data, we define the $|d|$ of Eq 1 from the Green's function which is defined in the main text. The momentum $(k)$ of the maximum of the momentum distribution curve (MDC) of the ARPES for each band (of index of $\nu$ ) are noted as $k_{\nu}^{M D C}$ assigned to each frequency of $\omega$ as shown in Eq S1. The quasiparticle spectra of $(\omega, k)$ is given by the condition of $\operatorname{det}\left(G^{-1}(k, \omega)\right)=0$ with the condition of $\operatorname{Im} \Sigma=0$. Thus, the variable $|d|$ should be minimized for each frequency. From the defined reference Hamiltonian of $H(k)$, the variable $|d|$ is a function of the self-energy for each frequency. We minimize $|d|$ as a root finding procedure from the self-energy variable for each frequency. From this procedure, the self-energy is extracted.

$$
\begin{gathered}
d^{2}=\sum_{\nu}\left|\operatorname{det}\left(G^{-1}\left(k_{\nu}^{M D C}, \omega\right)\right)\right|^{2} \\
\nu=(\alpha, \beta, \gamma, \text { and } \delta) \\
|d|=f\left(\Sigma_{x z / y z}(k, \omega), \Sigma_{x y}(k, \omega)\right)
\end{gathered}
$$

Several assumptions for the simplification of self-energy is made to solve Eq S1. The first, for the low energy, most of orbital character is $x z / y z$ and $x y$. Thus, we consider the self-energy of $x z / y z$ and $x y$ of $\operatorname{Fe}(d)$ only as shown in Eq S1. The second, we assume that self-energy is $k_{z}$ independent. The third, for the in-plane momentum dependence of the self-energy, we employed two ansätze, namely, the local $\Sigma$ ansatz and the non-local $\Sigma$ ansatz as shown in Eq $\underline{\text { S2 }}$

$$
\begin{aligned}
& \Sigma_{m}(k, \omega)=\Sigma_{m}(K, \omega) \text { non }- \text { local } \Sigma \text { ansatz } \\
& \Sigma_{m}(k, \omega)=\Sigma_{m}(\omega) \text { local } \Sigma \text { ansatz }
\end{aligned}
$$

In the local $\Sigma$ ansatz, the self-energy is momentum independent, and all bands of $\nu$ index for the given frequency is considered for the minimization of $|d|$ as shown in Eq S2. In the non-local $\Sigma$ ansatz, the self-energy is a coarse grained constant in the momentum space for $K$ of Brillouin zone (BZ) patch as shown in Eq S2. We used the 2 dimensional BZ patch in the main text, in relation to the existing antiferromagnetic (AFM) correlation with $q$ vector 
close to $M$. S50 In practice, the coarse grained momentum $K=\Gamma$ includes $k$ for $\alpha, \alpha^{\prime}$ and $\beta$, hole bands, and the coarse grained momentum $K=\mathrm{M}$ includes $k$ for $\delta$ and $\gamma$ electron bands. We consider $\alpha, \beta$, $\delta$, and $\gamma$ bands for the extraction of the momentum independent self-energies in the local $\Sigma$ ansatz. In the non-local $\Sigma$ ansatz, we consider $\alpha$ and $\beta$ bands for $K=\Gamma$, and $\delta$ and $\gamma$ bands for $K=\mathrm{M}$, for the extraction of the $K$ dependent self-energy as described in Eq S1. The description of $\alpha^{\prime}$ could be achieved from these procedures due to the similar $K$ dependence of self-energy in between $\alpha$ and $\alpha^{\prime}$ of $x z / y z$ orbital.

From the symmetry of the lattice of LiFeAs, we assumed that self-energy of $\mathrm{Fe}(d)$ is orbitally diagonal. From Refs. S46 S48, it is shown that for the regime of Hund's metal, due to the larger energy scale of Kondo screening in the orbital sector $\left(T_{K}^{o}\right)$ with respect to the $\mathrm{SOC}\left(\lambda_{S O C}\right), T_{K}^{o}>\lambda_{S O C}$, SOC is not effective on the orbitally diagonal self-energy having a frequency dependent coherence-incoherence crossover.

For orbitally off-diagonal self-energy, in Refs. $\mathrm{S} 46$ and $\underline{\mathrm{S} 47}$, it is shown that from the example of $\mathrm{Sr}_{2} \mathrm{RuO}_{4}$, this off-diagonal term could be absorbed into the renormalization of SOC. We assume that this orbitally off-diagonal self-energy is zero. We confirmed that this assumption properly describes the SOC effects on the ARPES near the zero frequency. Table S1 summarizes the effective SOC constant $\lambda_{S O C}$ of the reference Hamiltonian, and its comparison with the band splitting energy of $\alpha$ and $\alpha^{\prime}$ band at $k=\Gamma, \Delta$ for $\operatorname{ARPES}\left(\Delta_{A R P E S}\right), \operatorname{LDA}+\operatorname{SOC}\left(\Delta_{L D A}\right)$, and LQSGW+SOC $\left(\Delta_{L Q S G W}\right)$. The $\Delta$ is effectively equal to $Z \lambda_{S O C}(Z$ is the renormalization constant of the $x z / y z$ orbital at $k=\Gamma$ ) where $Z=1.0$ if the self-energy vanishes.

Table S1. The SOC induced splitting (in meV unit) of the $\alpha$ and $\alpha^{\prime}$ at $k=\Gamma, \Delta$, of ARPES $\left(\Delta_{A R P E S}\right)$ S56], LDA+SOC $\left(\Delta_{L D A}\right)$, and LQSGW+SOC $\left(\Delta_{L Q S G W}\right)$. We have shown the effective SOC $\left(\lambda_{S O C}\right)$ of the reference Hamiltonian, $H(k)$, of the LDA $\left(\lambda_{S O C}^{L D A}\right)$ and LQSGW $\left(\lambda_{S O C}^{L Q S G W}\right)$. The $\Delta$ of the LDA+non-local $\Sigma$ fit $\left(Z \lambda_{S O C}^{L D A}\right)$ and the LQSGW+non-local $\Sigma$ fit $\left(Z \lambda_{S O C}^{L Q S G W}\right)$ is also shown, to be compared with its experimental value of $\triangle_{A R P E S}$.

\begin{tabular}{||c|c|c|c|c|c|c||}
\hline$\Delta_{A R P E S}[\mathrm{~S} 56]$ & $\Delta_{L D A}$ & $\Delta_{L Q S G W}$ & $\lambda_{S O C}^{L D A}$ & $\lambda_{S O C}^{L Q S G W}$ & $Z \lambda_{S O C}^{L D A}$ & $Z \lambda_{S O C}^{L Q S G W}$ \\
\hline $9.5-11.4$ & 50 & 35 & 50 & 25 & 12.5 & 9.5 \\
\hline
\end{tabular}

\section{DETERMINATION OF $k_{z}$ FOR THE REFERENCE OF ELECTRON SHEETS FOR $H^{L D A}$ AND $H^{L Q S G W}$}

In this section, we describe the relation in between (i) the variable of $k_{z}$ for electron pockets for the reference Hamiltonian $H(k)$ and (ii) the spatial locality of self-energy and the validity of the Luttinger's theorem, as discussed in the main text. The Fermi momentum and the mass of the $x z / y z$ driven electron band $(\delta$ band) computed with both LDA and LQSGW reference Hamiltonians depend strongly on $k_{z}$ as shown in Fig S3, This in turns influences the extracted self-energy. Only one value of $k_{z}(0.55)$, together with the assumption of a $k_{z}$ independent self energy gives a three dimensional volume consistent with the Luttinger's theorem.

$L D A$ reference - Table $\mathbf{S 2}$ presents the self-energy from the LDA+non-local $\Sigma$ ansatz with $k_{z}=0.00$ for hole pockets and $k_{z}=0.55$ for electron pockets. It is shown that the self-energy of this case have a strong spatial non-locality with strong momentum $(\mathrm{K})$ dependence. This feature implies that the LDA+local $\Sigma$ ansatz is not applicable with $k_{z}=0.55$ for electron pockets. With this choice of $k_{z}=0.55$ for electron pockets, the LDA+non-local $\Sigma$ ansatz fulfills the Luttinger's theorem as shown in Table S3 and Fig S4(a). In Fig S4(a), it is shown that the LDA+non-local $\Sigma$ ansatz with $k_{z}=0.55$ for electron pockets provides an overestimation of total $k_{z}$ dependent dispersion of the $\delta$ Fermi surface with respect to the ARPES and the LQSGW+non-local $\Sigma$ ansatz with $k_{z}=0.55$ for electron pockets. S44] In the main text, we have shown that the setting of $k_{z}=0.35$ for electron pockets provides more local static self-energy $(\Sigma(0))$ but violates the Luttinger's theorem (see Table $\mathbf{S 3}$ and Table $\mathbf{\text { S5 }}$ ).

In summary, for the LDA reference, there is no $k_{z}$ for electron sheet such that fulfills both the locality of self-energy and the Luttinger's theorem together.

LQSGW reference - Table S4 presents the self-energy from the LQSGW+non-local $\Sigma$ ansatz with the $k_{z}=0.00$ for hole pockets and the $k_{z}=0.35$ for electron pockets. It is shown that the self-energy of this case have a strong spatial non-locality with strong momentum $(\mathrm{K})$ dependence. With this choice of the $k_{z}=0.35$ for electron pockets, the LQSGW+non-local $\Sigma$ ansatz violates the Luttinger's theorem as shown in Table S3 and Fig S4(b). In the main text, we have shown that for the setting of $k_{z}=0.55$ for electron pockets, the Luttinger's theorem is obeyed and the self-energy is spatially local (see Table S3 and Table S6).

In summary, for the LQSGW reference, there is single value of $k_{z}=0.55$ for electron pockets which (i) fulfills the Luttinger's theorem and (ii) provides a validity of the spatially local self-energy. 

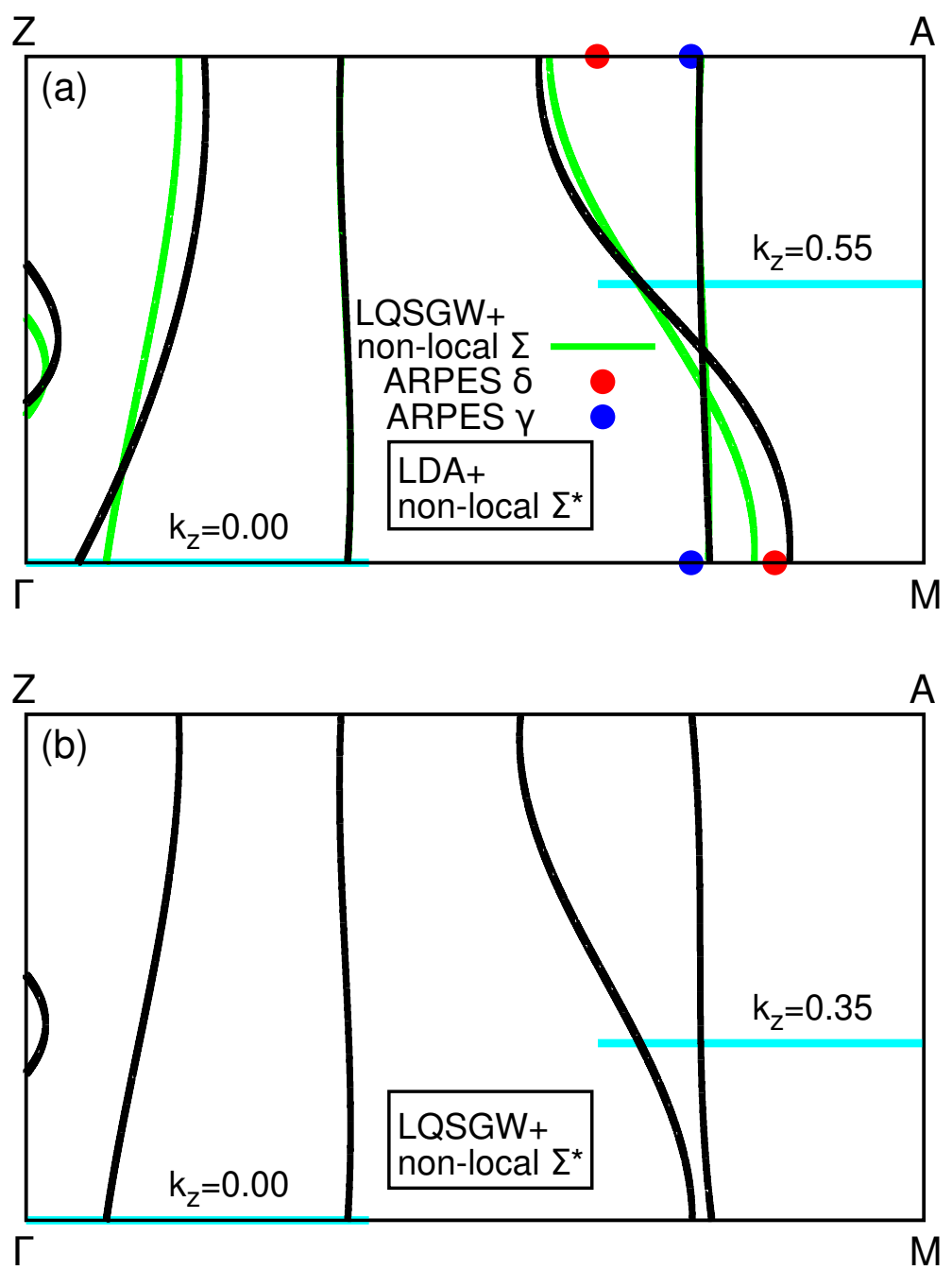

Figure S4. (a) Fermi surfaces for the LDA+non-local $\Sigma$ ansatz with $k_{z}=0.00$ for hole bands fit and $k_{z}=0.55$ for electron bands fit (black color) (total Fermi surface volume of -0.01 (electrons/unit cell)). Red and blue dots are the $\delta$ and $\gamma$ Fermi surfaces measured with ARPES in Ref.[S44]. Green lines are for the Fermi surface of the LQSGW+non-local $\Sigma$ ansatz with $k_{z}=0.00$ for hole bands fit and $k_{z}=0.55$ for electron bands fit (b) Fermi surfaces for the LQSGW+non-local $\Sigma$ ansatz with $k_{z}=0.00$ for hole bands fit and $k_{z}=0.35$ for electron bands fit (total Fermi surface volume of +0.18 (electrons/unit cell)). The ARPES data for fitting is taken from Ref. [S20] (See main text).

Table S2. Zero frequency self-energy $\left(\Sigma_{m}(K, 0)\right)$ and quasiparticle residue $\left(\mathrm{Z}_{m}(\mathrm{~K})\right)$ from the ARPES of LiFeAs with the LDA reference using the non-local $\Sigma$ ansatz. We set $k_{z}=0.00$ for $\mathrm{K}=\Gamma$ (for hole sheets) and $k_{z}=0.55$ for $\mathrm{K}=\mathrm{M}$ (for electron sheets), for the LDA reference. Error bars (total) are computed from the peak width of both in plane $k$ and out of plane $k_{z}$. For the peak width, we adapted the resolution in the MDC of LiFeAs, $0.01(\pi / a)\left(0.008 \AA^{-1}\right)$. Here, $\sqrt{2} a$ is the $a_{\text {lattice }}$, where $a_{\text {lattice }}$ is the lattice constant of LiFeAs. This resolution in the MDC is taken from the best resolution limit for the $\beta$ sheet with the resolution limit of the energy of $3 \mathrm{meV}$.

\begin{tabular}{||c||c|c|c|c||}
\hline & $\Sigma_{m}(\Gamma, 0)(\mathrm{eV})$ & $\mathrm{Z}_{m}(\Gamma)$ & $\Sigma_{m}(M, 0)(\mathrm{eV})$ & $\mathrm{Z}_{m}(\mathrm{M})$ \\
\hline$x y$ & $0.029 \pm 0.025$ & $0.15 \pm 0.01$ & $-0.153 \pm 0.060$ & $0.10 \pm 0.01$ \\
\hline$x z / y z$ & $-0.083 \pm 0.040$ & $0.25 \pm 0.13$ & $0.042 \pm 0.014$ & $0.34 \pm 0.04$ \\
\hline
\end{tabular}

\section{ERROR BAR IN THE SELF-ENERGY, IN PLANE $k$ ERROR VERSUS OUT OF PLANE $k_{z}$ ERROR}

In this section, we describe the procedure for the extraction of the error bar in the self-energy.

In the extraction of error bar of zero frequency self-energy $\left(\Sigma_{m}(K, 0)\right)$ and quasiparticle residue $\left(\mathrm{Z}_{m}(\mathrm{~K})\right)$ from the ARPES, we consider two independent sources of error (i) in plane $k$ error and (ii) out of plane $k_{z}$ error, with consideration of the MDC peak width of $0.01(\pi / a)\left(0.008 \AA^{-1}\right)$. There is an intrinsic uncertainty in the value of $k_{z}$ 
Table S3. The Fermi surface volumes (electrons/unit cell) in (a) the LDA, (b) the non-local $\Sigma$ fitting with the reference of the LDA with $k_{z}=0.35$ for electron bands, (c) the local $\Sigma$ fitting with the reference of the LDA with $k_{z}=0.35$ for electron bands, (d) the non-local $\Sigma$ fitting with the reference of the LDA with $k_{z}=0.55$ for electron bands (See Fig[S4(a)), (e) the LQSGW, (f) the non-local $\Sigma$ fitting with the reference of LQSGW with $k_{z}=0.55$ for electron bands, and (g) the results of the LQSGW+non-local $\Sigma$ fit with $k_{z}=0.35$ for electron bands which violates the Luttinger's theorem (See Fig S4 (b)). We fit the hole bands with $k_{z}=0.00$ for both the LDA and the LQSGW references.

\begin{tabular}{||c||c|c|c|c|c|c||}
\hline & $\alpha$ & $\alpha$ & $\beta$ & $\gamma$ & $\delta$ & total \\
\hline LDA & 0.01 & 0.14 & 0.33 & 0.18 & 0.28 & -0.02 \\
\hline LDA+non-local $\Sigma\left(k_{z}=0.35\right.$ for fitting of electron bands $)$ & 0.00 & 0.08 & 0.37 & 0.22 & 0.39 & +0.17 \\
\hline LDA+local $\Sigma\left(k_{z}=0.35\right.$ for fitting of electron bands $)$ & 0.00 & 0.06 & 0.36 & 0.19 & 0.35 & +0.12 \\
\hline LDA+non-local $\Sigma\left(k_{z}=0.55\right.$ for fitting of electron bands $)$ & 0.00 & 0.08 & 0.37 & 0.19 & 0.24 & -0.01 \\
\hline LQSGW & 0.00 & 0.08 & 0.35 & 0.20 & 0.21 & -0.03 \\
\hline LQSGW+non-local $\Sigma\left(k_{z}=0.55\right.$ for fitting of electron bands $)$ & 0.00 & 0.05 & 0.36 & 0.20 & 0.26 & +0.04 \\
\hline LQSGW + non-local $\Sigma\left(k_{z}=0.35\right.$ for fitting of electron bands $)$ & 0.00 & 0.05 & 0.36 & 0.23 & 0.37 & +0.18 \\
\hline
\end{tabular}

Table S4. Zero frequency self-energy $\left(\Sigma_{m}(K, 0)\right)$ and quasiparticle residue $\left(\mathrm{Z}_{m}(\mathrm{~K})\right)$ from the ARPES of LiFeAs with the LQSGW reference using the non-local $\Sigma$ ansatz. We set $k_{z}=0.00$ for $\mathrm{K}=\Gamma$ (for hole sheets) and $k_{z}=0.35$ for $\mathrm{K}=\mathrm{M}$ (for electron sheets), for the LQSGW reference. Error bars (total) are computed from the peak width of both in plane $k$ and out of plane $k_{z}$. For the peak width, we adapted the resolution in the MDC of LiFeAs, $0.01(\pi / a)\left(0.008 \AA^{-1}\right)$. Here, $\sqrt{2} a$ is the $a_{\text {lattice }}$, where $a_{\text {lattice }}$ is the lattice constant of LiFeAs. This resolution in the MDC is taken from the best resolution limit for the $\beta$ sheet with the resolution limit of the energy of $3 \mathrm{meV}$.

\begin{tabular}{||c||c|c|c|c||}
\hline & $\Sigma_{m}(\Gamma, 0)(\mathrm{eV})$ & $\mathrm{Z}_{m}(\Gamma)$ & $\Sigma_{m}(M, 0)(\mathrm{eV})$ & $\mathrm{Z}_{m}(\mathrm{M})$ \\
\hline$x y$ & $0.002 \pm 0.014$ & $0.21 \pm 0.01$ & $0.041 \pm 0.033$ & $0.21 \pm 0.01$ \\
\hline$x z / y z$ & $-0.027 \pm 0.003$ & $0.38 \pm 0.01$ & $-0.188 \pm 0.021$ & $0.16 \pm 0.01$ \\
\hline
\end{tabular}

Table S5. Zero frequency self-energy $\left(\Sigma_{m}(K, 0)\right)$ and quasiparticle residue $\left(\mathrm{Z}_{m}(\mathrm{~K})\right)$ from the ARPES of LiFeAs with the LDA reference using the non-local $\Sigma$ ansatz. We set $k_{z}=0.00$ for $\mathrm{K}=\Gamma$ (for hole sheets) and $k_{z}=0.35$ for $\mathrm{K}=\mathrm{M}$ (for electron sheets), for the LDA reference. Error bars are computed from the peak width of in-plane $k$ (parentheses is out of plane $k_{z}$ ). For the peak width, we adapted the resolution in the MDC of LiFeAs, $0.01(\pi / a)\left(0.008 \AA^{-1}\right)$. Here, $\sqrt{2} a$ is the $a_{\text {lattice }}$, where $a_{\text {lattice }}$ is the lattice constant of LiFeAs. This resolution in the MDC is taken from the best resolution limit for the $\beta$ sheet with the resolution limit of the energy of $3 \mathrm{meV}$.

\begin{tabular}{||c||c|c|c|c||}
\hline & $\Sigma_{m}(\Gamma, 0)(\mathrm{eV})$ & $\mathrm{Z}_{m}(\Gamma)$ & $\Sigma_{m}(M, 0)(\mathrm{eV})$ & $\mathrm{Z}_{m}(\mathrm{M})$ \\
\hline$x y$ & $0.029 \pm 0.025(0.000)$ & $0.15 \pm 0.01(0.00)$ & $-0.130 \pm 0.062(0.004)$ & $0.12 \pm 0.01(0.01)$ \\
\hline$x z / y z$ & $-0.083 \pm 0.040(0.000)$ & $0.25 \pm 0.13(0.01)$ & $-0.113 \pm 0.013(0.023)$ & $0.16 \pm 0.01(0.02)$ \\
\hline
\end{tabular}

Table S6. Zero frequency self-energy $\left(\Sigma_{m}(K, 0)\right)$ and quasiparticle residue $\left(\mathrm{Z}_{m}(\mathrm{~K})\right)$ from the ARPES of LiFeAs with the LQSGW reference using the non-local $\Sigma$ ansatz. We set $k_{z}=0.00$ for $\mathrm{K}=\Gamma$ (for hole sheets) and $k_{z}=0.55$ for $\mathrm{K}=\mathrm{M}$ (for electron sheets), for the LQSGW reference. Error bars are computed from the peak width of in-plane $k$ (parentheses is out of plane $\left.k_{z}\right)$. For the peak width, we adapted the resolution in the MDC of LiFeAs, $0.01(\pi / a)\left(0.008 \AA^{-1}\right)$. Here, $\sqrt{2} a$ is the $a_{\text {lattice }}$, where $a_{\text {lattice }}$ is the lattice constant of LiFeAs. This resolution in the MDC is taken from the best resolution limit for the $\beta$ sheet with the resolution limit of the energy of $3 \mathrm{meV}$.

\begin{tabular}{||c||c|c|c|c||}
\hline & $\Sigma_{m}(\Gamma, 0)(\mathrm{eV})$ & $\mathrm{Z}_{m}(\Gamma)$ & $\Sigma_{m}(M, 0)(\mathrm{eV})$ & $\mathrm{Z}_{m}(\mathrm{M})$ \\
\hline$x y$ & $0.002 \pm 0.014(0.000)$ & $0.21 \pm 0.01(0.00)$ & $0.044 \pm 0.036(0.001)$ & $0.18 \pm 0.00(0.01)$ \\
\hline$x z / y z$ & $-0.027 \pm 0.003(0.000)$ & $0.38 \pm 0.01(0.00)$ & $-0.051 \pm 0.006(0.114)$ & $0.30 \pm 0.03(0.03)$ \\
\hline
\end{tabular}

as the surface breaks translation symmetry and therefore, the $k_{z}$ is not a good quantum number. As shown in Fig 3 , the most dominant $k_{z}$ dependent variation is in the $x z / y z$ orbital dominant $\delta$ band placed at $K=\mathrm{M}$, and other bands have a smaller $k_{z}$ dependency. As a results, the most of the error bars are from the in plane $k$ error, and a sizable $k_{z}$ peak width induced error is only at the $K=\mathrm{M}$ for $x z / y z$ orbital (contribute to the $\delta$ band), for both the LDA reference and the LQSGW reference, as shown in Table S5 and Table S6. The large Fermi velocity of the $\gamma$ band results in the large error bar in the zero frequency self-energy of the $x y$ orbital at $K=\mathrm{M}$ from the in plane $k$ error. 


\section{LOCALITY OF DYNAMICAL SELF-ENERGY}

In this section, we present dynamical part self-energies in the LDA reference and LQSGW reference, in the main text, with $k_{z}=0.35$ for electron bands fit in the LDA reference and $k_{z}=0.55$ for electron bands fit in the LQSGW reference.

The full frequency dependence of the extracted self-energies is presented on Fig. S5, which displays the dynamical part $\operatorname{Re} \Sigma_{m}(K, \omega)-\operatorname{Re} \Sigma_{m}(K, 0)$ for the different schemes considered in this work. By comparing the $K=\Gamma$ and $K=M$ data, it is immediately apparent from this figure that the fits based on the LQSGW reference lead to a much higher degree of locality for both orbital components than those based on the LDA reference. These factors imply that the self-energy from the LQSGW referencce is spatially local, confirms the validity of the LQSGW+local $\Sigma$ ansatz for the description of the quasiparticle of LiFeAs.
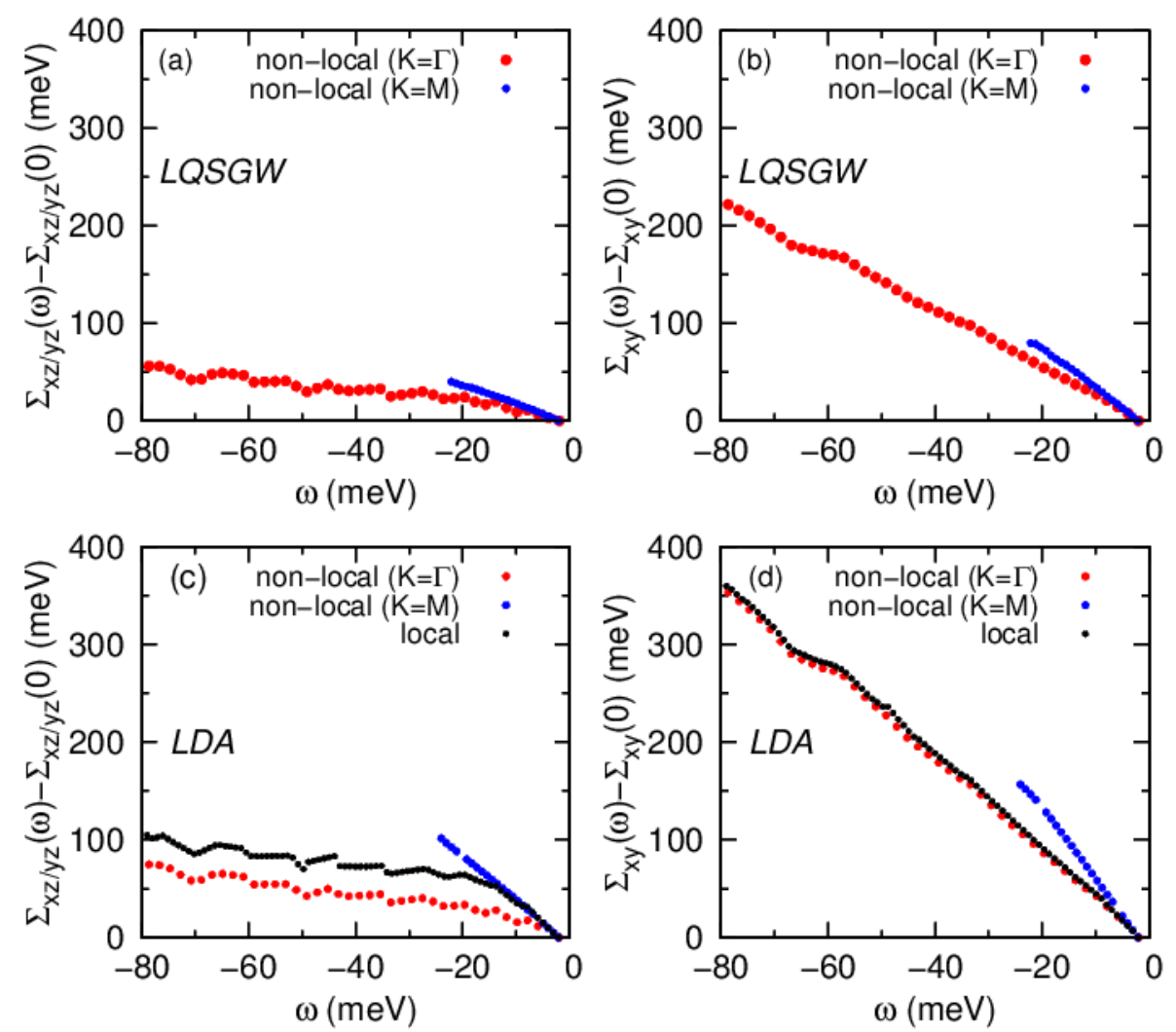

Figure S5. (a) and (b) Dynamical part of the self energies, $\Sigma_{m}(K, \omega)-\Sigma_{m}(K, 0)$ in the LQSGW+non-local $\Sigma$ ansatz for $x z / y z$ orbital and $x y$ orbital, respectively. (c) and (d) Dynamical part self energy of $x z / y z$ and $x y$ orbitals, in the LDA+non-local $\Sigma$ ansatz and in the LDA+local $\Sigma$ ansatz. Here, we use $k_{z}=0.00$ for hole bands for both the LDA and the LQSGW references. We use $k_{z}=0.35$ for electron bands for the LDA references. We use $k_{z}=0.55$ for electron bands for the LQSGW references (see the main text and the Section V).

\section{VALIDITY OF THE LQSGW+NON-LOCAL $\Sigma$ ANSATZ FOR ARPES}

To show the validity of the extracted self-energy of the LQSGW+non-local $\Sigma$ ansatz from the quasiparticle of the ARPES experiment in the main text, we compared spectra from the LQSGW+non-local $\Sigma$ ansatz to the ARPES of Ref. [S20] in Fig[S6 and the ARPES of Ref. [S54] in Fig [S7] with different $k$ path $(\Gamma-M)$ and $k_{z}$ values. Fig[S6] and Fig S7 implies that present LQSGW+non-local $\Sigma$ ansatz provides a good description of the quasiparticle spectra for multiple examples of the $k$ path and the $k_{z}$. 

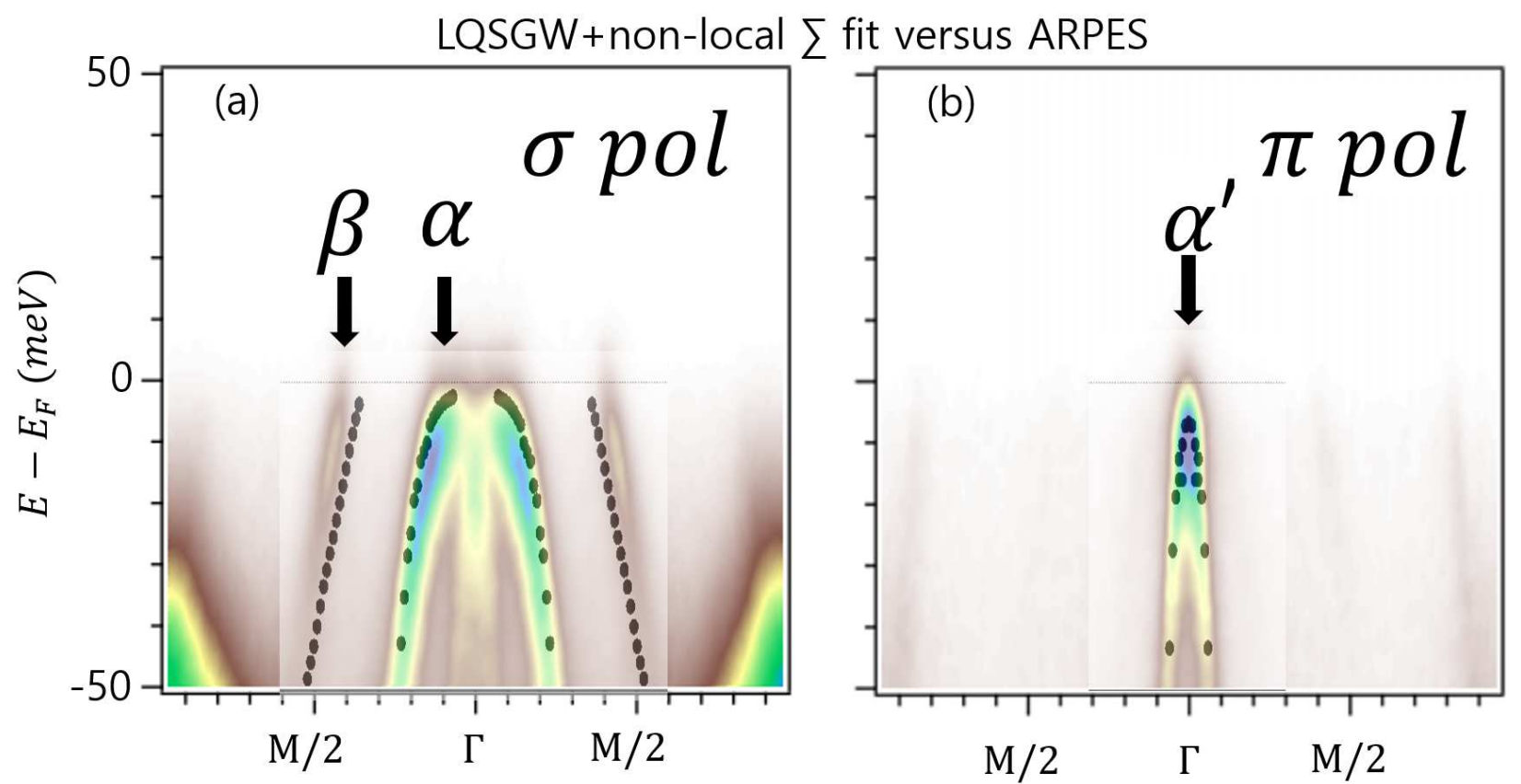

Figure S6. (a) Comparison of band dispersions of LiFeAs of Ref. S20] from the ARPES (color contour) and the LQSGW+nonlocal $\Sigma$ ansatz for hole pockets $(\alpha$ and $\beta)$ in the $\Gamma$-M $k$ path $\left(k_{z}=0.00\right)$ from $\sigma$ polarized light. (b) Same as (a) for $\alpha^{\prime}$ from $\pi$ polarized light. $[\mathrm{S} 20]$

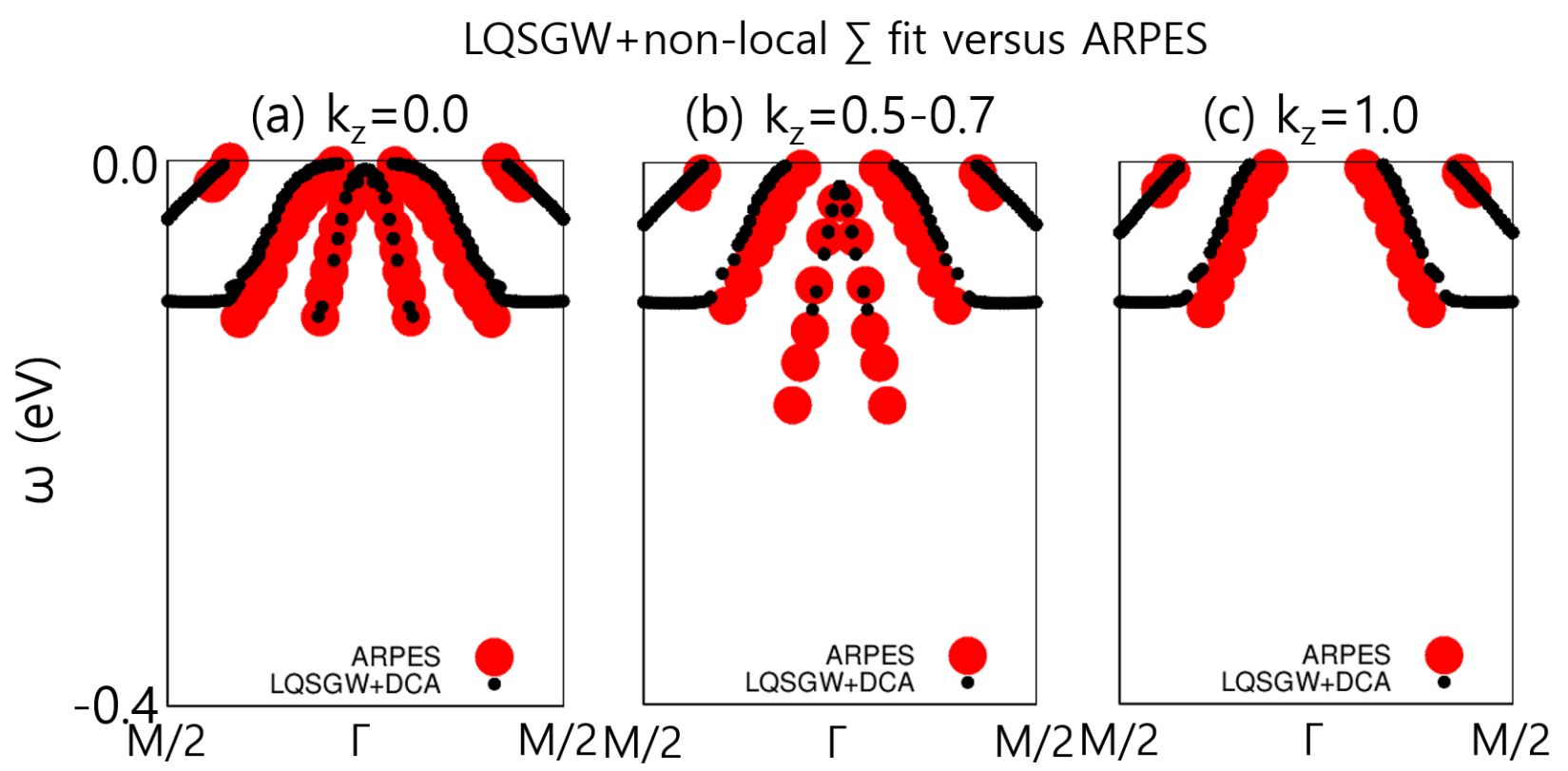

Figure S7. (a) Comparison of band dispersions of LiFeAs of Ref. [S54] from the ARPES (red circle) and the LQSGW+nonlocal $\Sigma$ ansatz (black dots) for $k_{z}=0.00$ for hole pockets $\left(\alpha, \alpha^{\prime}\right.$, and $\beta$ ) in the $\Gamma$-M $k$ path $\left(k_{z}=0.00\right)$. (b) Same as (a) for $k_{z}=0.50-0.70$ S54]. (c) Same as (a) for $k_{z}=1.00[\mathrm{~S} 54]$.

\section{SPIN ORBIT COUPLING OF LIFEAS}

In this section we estimate the admixture of $x z / y z$ and $x y$ orbital character near the Fermi level near the $\Gamma$ point.

Fig $\$ 8$ presents single particle $t_{2 g}$ states in the presence of a tetragonal crystal field $\left(\varepsilon_{t}\right)$ and SOC $(\lambda)$ as defined by the one site Hamiltonian in Eq $\underline{\text { S3. }}$. 
(a) strong tetragonal crystal field

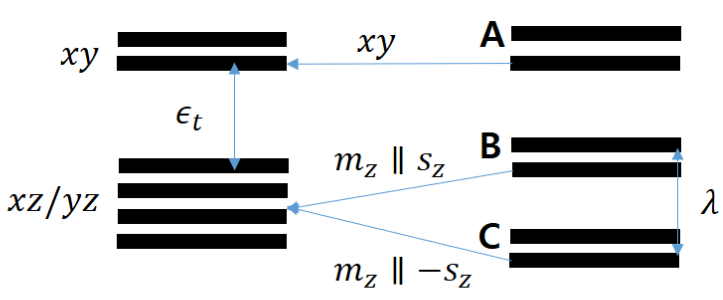

(b) strong SOC

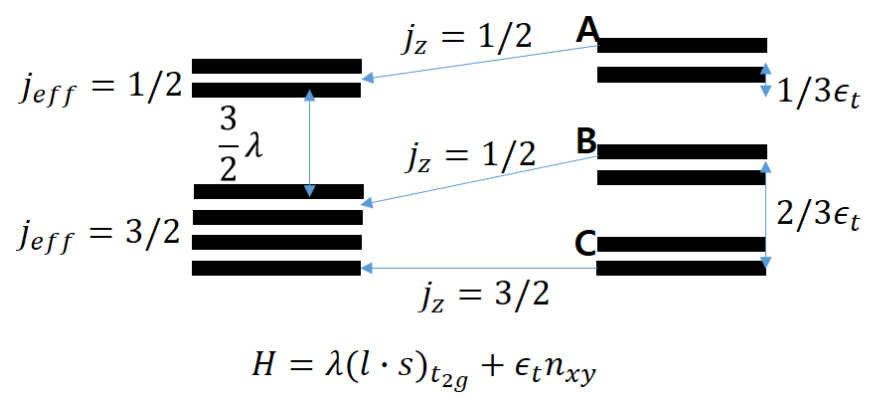

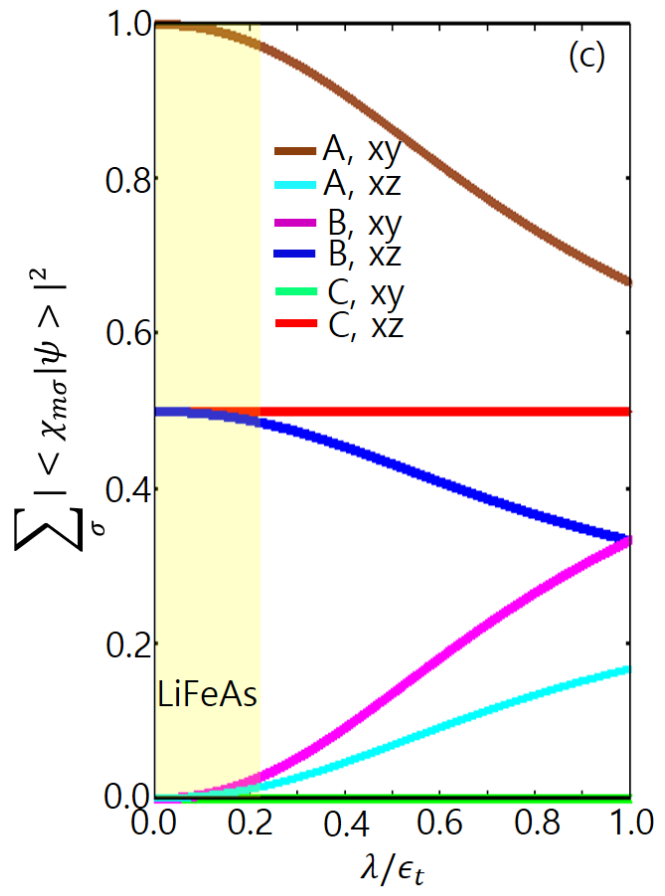

Figure S8. (a) Multiplet states in the presence of strong tetragonal crystal field $\left(\varepsilon_{t}\right)$, with small spin orbit coupling $(\lambda)$. (b) Multiplet states in the presence of strong spin orbit coupling $(\lambda)$ small tetragonal crystal field $\left(\varepsilon_{t}\right)$. A, B, and C Kramers doublets are continuously connected from the regime of (a) to the regime of (b), presented in Eq S5 and Eq[S6 (c) Orbital contents of A, B, and C Kramers doublet with variation of $\lambda / \varepsilon_{t} . \psi$ is the Kramer's doublet A, B, and C of Eq S5 and Eq S6. $\chi_{m, \sigma}$ is the atomic $t_{2 g}$ orbital ( $m$ : orbital, $\sigma: \operatorname{spin}$ ). From the tetragonal symmetry, the $y z$ orbital have a same projection weight with respect to the $x z$ orbital. The regime for non-degenerate bands of LiFeAs is denoted from yellow color.

$$
H=\lambda(l \cdot s)_{t_{2 g}}+\frac{1}{2} \varepsilon_{t}\left(c_{x y, \uparrow}^{\dagger} c_{x y, \uparrow}+c_{x y, \downarrow}^{\dagger} c_{x y, \downarrow}-c_{x z, \uparrow}^{\dagger} c_{x z, \uparrow}-c_{x z, \downarrow}^{\dagger} c_{x z, \downarrow}-c_{y z, \uparrow}^{\dagger} c_{y z, \uparrow}-c_{y z, \downarrow}^{\dagger} c_{y z, \downarrow}\right)
$$

The $\lambda(l \cdot s)_{t_{2 g}}$ of Eq $[3$ is given by Eq $[\mathbf{S 4}$ in the order of $x z, \uparrow, y z, \uparrow, x y, \uparrow, x z, \downarrow, y z, \downarrow$, and $x y, \downarrow$ states.

$$
\lambda(l \cdot s)_{t_{2 g}}=\left(\begin{array}{cccccc}
0 & -i \frac{\lambda}{2} & 0 & 0 & 0 & i \frac{\lambda}{2} \\
i \frac{\lambda}{2} & 0 & 0 & 0 & 0 & -\frac{\lambda}{2} \\
0 & 0 & 0 & -i \frac{\lambda}{2} & \frac{\lambda}{2} & 0 \\
0 & 0 & i \frac{\lambda}{2} & 0 & i \frac{\lambda}{2} & 0 \\
0 & 0 & \frac{\lambda}{2} & -i \frac{\lambda}{2} & 0 & 0 \\
-i \frac{\lambda}{2} & -\frac{\lambda}{2} & 0 & 0 & 0 & 0
\end{array}\right)
$$

The strong and weak $\lambda$ cases are shown in Fig $\underline{S 8}(\mathrm{~b})$ and Fig $\mathrm{S8}(\mathrm{a})$, respectively. The eigenvectors of the Hamiltonian Eq $\left[\right.$ S3, are Kramer's doublets A, B, and C. Their orbital content is shown schematically as a function of $\frac{\lambda}{\varepsilon_{t}}$ in Fig 


$$
\begin{aligned}
& \left|m_{z}\right| \mid s_{z}, s_{z}=\uparrow>=-\frac{1}{\sqrt{2}}(|x z, \uparrow>+i| y z, \uparrow>) \\
& \left|m_{z}\right| \mid s_{z}, s_{z} \downarrow>=\frac{1}{\sqrt{2}}(|x z, \downarrow>-i| y z, \downarrow) \\
& \left|m_{z}\right| \mid-s_{z}, s_{z}=\downarrow>=-\frac{1}{\sqrt{2}}(|x z, \downarrow>+i| y z, \downarrow>) \\
& \left|m_{z}\right| \mid-s_{z}, s_{z}=\uparrow>=\frac{1}{\sqrt{2}}(|x z, \uparrow>-i| y z, \uparrow) \\
& \mid x y, \uparrow> \\
& \mid x y, \downarrow> \\
& \mid j_{\text {eff }}=1 / 2, j_{z}=1 / 2> \\
& =-\frac{1}{\sqrt{3}}(|x y, \uparrow>+| y z, \downarrow>+i \mid x z, \downarrow>) \\
& \mid j_{\text {eff }}=1 / 2, j_{z}=-1 / 2> \\
& =\frac{1}{\sqrt{3}}(|x y, \downarrow>-| y z, \uparrow>+i \mid x z, \uparrow>) \\
& \mid j_{\text {eff }}=3 / 2, j_{z}=1 / 2> \\
& =\frac{1}{\sqrt{6}}(2|x y, \uparrow>-| y z, \downarrow>-i \mid x z, \downarrow>) \\
& \mid j_{\text {eff }}=3 / 2, j_{z}=-1 / 2> \\
& =\frac{1}{\sqrt{6}}(2|x y, \downarrow>+| y z, \uparrow>-i \mid x z, \uparrow>) \\
& \mid j_{\text {eff }}=3 / 2, j_{z}=3 / 2> \\
& =-\frac{1}{\sqrt{2}}(|y z, \uparrow>+i| x z, \uparrow>) \\
& \mid j_{e f f}=3 / 2, j_{z}=-3 / 2> \\
& =\frac{1}{\sqrt{2}}(|y z, \downarrow>-i| x z, \downarrow>)
\end{aligned}
$$

The single particle states A, B, and C of Eq 55 and Eq $\left[\right.$ is connected with the variable of $\lambda / \varepsilon_{t}$. Fig $\mathbf{S 8}$ (c) presents orbital contents in the $t_{2 g}$ orbital in the presence of the tetragonal crystal field $\left(\varepsilon_{t}\right)$ and SOC $(\lambda)$, as a function of $\lambda / \varepsilon_{t}$.

In the region of parameters that correspond to the $\Gamma$ point of LiFeAs, $\varepsilon_{t}$ correspond to $\sim 250 \mathrm{meV} \lambda_{S O C}=50 \mathrm{meV}$, within LDA, hence the ratio $\lambda_{S O C} / \varepsilon_{t}$ is less than 0.2 for LiFeAs. This estimate of the upper limit of $\lambda_{S O C} / \varepsilon_{t}$ also holds for LQSGW $+\lambda_{S O C}$.

\section{TRANSPORT PROPERTIES OF LIFEAS AND THE LQSGW+LOCAL $\Sigma$ ANSATZ}

In this section, we analyze transport data to show that a spatially local scattering rate of LiFeAs, motivated by the LQSGW+local $\Sigma$ ansatz, provides a natural explanation for the transport experiments of Ref. S59. We can view this as an independent corroboration, that the scattering rate is spatially local, while strongly orbital dependent can be taken to be the same in the electron and hole pockets.

Fig $S 9$ presents the experimental temperature dependent resistivity $(\rho)$ and the experimental temperature dependent Hall coefficient adapted from Ref. [S59]. This data shows that the Hall coefficient is electron like, most prominently around the temperature of $\sim 100 \mathrm{~K}$. With lowering of the temperature below $\sim 100 \mathrm{~K}$, this electron like Hall conductivity is reduced. Around the temperature of $\sim 100 \mathrm{~K}$, the resistivity data shows that upon cooling from this temperature, a Fermi liquid behaviour is obtained as $\rho$ proportional to $T^{2}$. 

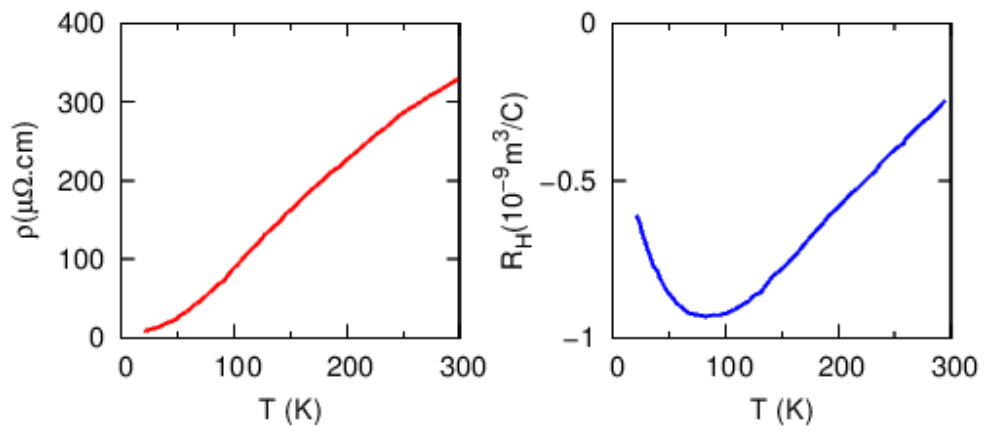

Figure S9. Experimental temperature dependent resistivity $(\rho)$ of LiFeAs (left panel), and experimental temperature dependent Hall coefficient $\left(\mathrm{R}_{H}\right)$ of LiFeAs (right panel), adapted from Ref. $[\mathrm{S} 59]$.

Table S7. Mass of the bare band structure $\left(m_{\nu}, \nu=\alpha, \beta, \gamma\right.$, and $\left.\delta\right)$ of LQSGW $+\lambda_{S O C}$ in the unit of the free electron mass $\left(m_{e}\right)$, and the charge carrier number $\left(n_{\nu}\right)$ of the LQSGW+non-local $\Sigma$ ansatz from Table $\$ 3$ ( $k_{z}=0.55$ for electron bands fit).

\begin{tabular}{||c|c|c|c||}
\hline \hline$m_{\alpha}$ & $m_{\beta}$ & $m_{\gamma}$ & $m_{\delta}$ \\
\hline $1.73 m_{e}$ & $1.36 m_{e}$ & $0.79 m_{e}$ & $1.20 m_{e}$ \\
\hline \hline$n_{\alpha}$ & $n_{\beta}$ & $n_{\gamma}$ & $n_{\delta}$ \\
\hline 0.05 & 0.36 & 0.20 & 0.26 \\
\hline \hline
\end{tabular}

We use a very simplified model of the transport summarized in Eq S7 expressing the resistivity and the Hall coefficient in terms of the mobility $\mu$, a charge carrier number $n$ and a scattering rate $(1 / \tau)$ with the mobility $\mu$, of each FS pocket given by EqS8.

Assuming that the scattering rate $(1 / \tau)$ depends on the orbital but not on whether the pocket is electron or hole like, we apply the scattering rate, $(1 / \tau)$, of the $x z / y z$ to $\alpha$ and $\delta$ Fermi surfaces, while the scattering rate of the $x y$ is applied to $\beta$ and $\gamma$ Fermi surfaces. $m_{\nu}$ is the bare mass of each FS which we estimate from our LQSGW reference as shown in Table S7. $m_{\alpha}$ and $m_{\beta}$ are estimated from the $\Gamma$-X $k$-path $\left(k_{z}=0.00\right)$, while $m_{\gamma}$ is estimated from $\Gamma$ M $k$-path $\left(k_{z}=0.55\right)$. While $m_{\delta}$ has substantial $k_{z}$ dependence as shown in Fig S3, transport quantities involve a weighted average of occupation and mobility. In our simplified model, we use an average value $1.20 m_{e}$ as summarized in Table S7.

We checked that variations in $m_{\delta}$ in the range of $\left[1.00 m_{e}, 1.80 m_{e}\right]$ gives rise to similar trends for the extracted scattering rate, and the converted transport data.

For the carrier number of each FS, we take the value from the LQSGW+non-local $\Sigma$ ansatz as shown in Table $\mathbf{S 7}$.

$$
\begin{aligned}
& \rho^{-1}(T)=\sum_{\nu} n_{\nu} e \mu_{\nu} \\
& R_{H}(T)=\left(\sum_{\nu} n_{\nu} e \mu_{\nu}^{2} \times \operatorname{sgn}(\nu)\right) / \sigma^{2}, \operatorname{sgn}(\nu)=+1 \text { for holes, }-1 \text { for electrons }
\end{aligned}
$$

$$
\begin{aligned}
& \mu_{\alpha}=\frac{e \tau_{x z / y z}}{m_{\alpha}} \\
& \mu_{\beta}=\frac{e \tau_{x y}}{m_{\beta}} \\
& \mu_{\gamma}=\frac{e \tau_{x y}}{m_{\gamma}} \\
& \mu_{\delta}=\frac{e \tau_{x z / y z}}{m_{\delta}}
\end{aligned}
$$

Fig $\$ 10$ presents extracted temperature dependent scattering rate $(1 / \tau)$ of the $x z / y z$ and the $x y$ orbitals from the experimental data of $\rho$ and $\mathrm{R}_{H}$ of Ref. S59]. It is shown that the $x z / y z$ orbital is more coherent than the $x y$ orbital. 


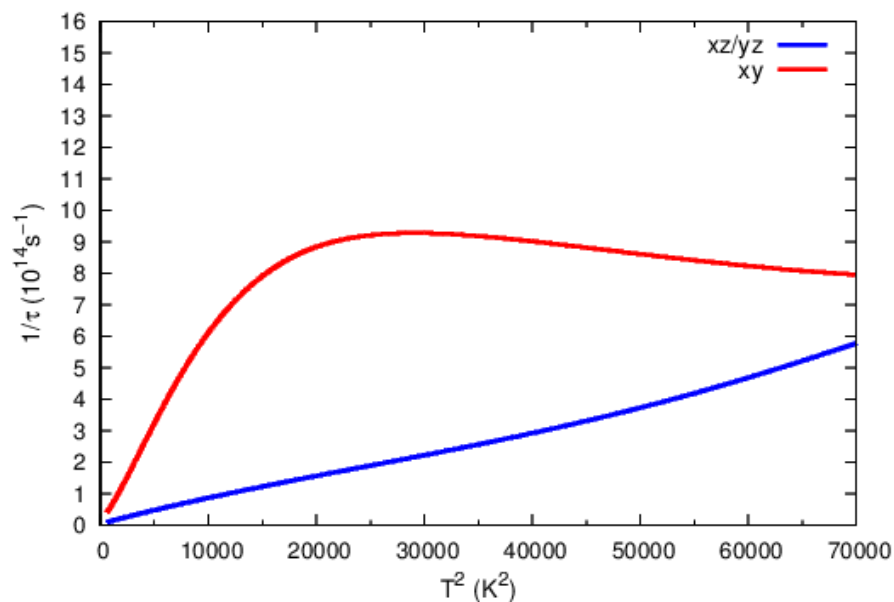

Figure S10. Extracted scattering rate $(1 / \tau)$ of $x z / y z$ and $x y$ orbitals from the data of Fig [S9 S59] and using Eq S7. Eq[S8. and Table S7.

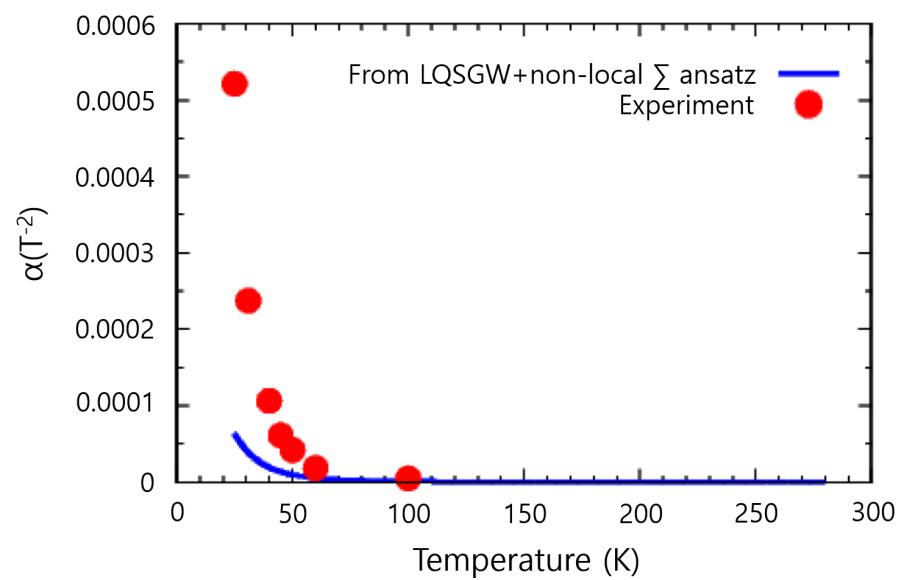

Figure S11. Computed temperature dependent magnetoresistance coefficient $(\alpha)$ from Eq $\mathbf{S 9}$ and Eq $\mathbf{S 8}$ using the extracted scattering rate $1 / \tau$ of Fig 10 with Table S7 of the LQSGW+non-local $\Sigma$ ansatz (blue line). The computed $\alpha$ is compared with its experimental value adapted from Ref. $[$ 559] (red dots).

The scattering rate of the $x z / y z$ orbital is nearly proportional to $T^{2}$ from $0 \mathrm{~K}\left(0 \mathrm{~K}^{2}\right)$ to $265 \mathrm{~K}\left(70000 \mathrm{~K}^{2}\right)$, implying that a Fermi liquid behaviour for this temperature range. The scattering rate of the $x y$ orbital is nearly proportional to $T^{2}$ from $0 \mathrm{~K}\left(0 \mathrm{~K}^{2}\right)$ to $100 \mathrm{~K}\left(10000 \mathrm{~K}^{2}\right)$, and there is a coherence-incoherence crossover around the temperature of $150 \mathrm{~K}$. This result of the scattering rate (Fig S10 from the transport data of Fig S9 implies that (i) the $x y$ orbital is more incoherent than the $x z / y z$ orbital, and (ii) there is no anomalous behaviour for the $x z / y z$ orbital.

To check the validity of the orbital dependent spatially local scattering rate in Fig $[\mathbf{S 1 0}$ we compute magnetoresistance (MR) coefficient, $\alpha$, from the scattering rate using the formulation of Eq S9. Fig $\mathbf{S 1 1}$ compares the computed MR coefficient with its experimental value of Ref. S59]. It is shown that the computed MR coefficient is consistent with the experimental MR coefficient, validates the local ansatz for the orbital dependent scattering rate. 


$$
\begin{gathered}
\alpha(T)=\frac{\delta \rho(T, H)}{\rho(T, 0) H^{2}} \\
\frac{\delta \rho(T, H)}{\rho(T, 0)}=-\frac{\delta \sigma(T, H)}{\sigma(T, 0)}-\left(\frac{\sigma_{x y}}{\sigma_{x x}}\right)^{2} \\
\sigma_{x y}=\quad H \sigma_{x x}^{2} R_{H} \\
\left(\frac{\sigma_{x y}}{\sigma_{x x}}\right)^{2}=H^{2} \sigma_{x x}^{2} R_{H}^{2}=H^{2} \sigma^{2} R_{H}^{2} \\
-\frac{\delta \sigma(T, H)}{\sigma(T, 0)}=\left[\left(\sum_{\nu} n_{\nu} e \mu_{\nu}^{3}\right) / \sigma\right] H^{2}
\end{gathered}
$$

garix.minjae.kim@gmail.com

[S1] Y. Kamihara, H. Hiramatsu, M. Hirano, R. Kawamura, H. Yanagi, T. Kamiya, and H. Hosono, Journal of the American Chemical Society 128, 10012 (2006).

[S2] I. Mazin, D. J. Singh, M. Johannes, and M.-H. Du, Physical Review Letters 101, 057003 (2008).

[S3] K. Kuroki, S. Onari, R. Arita, H. Usui, Y. Tanaka, H. Kontani, and H. Aoki, Physical Review Letters 101, 087004 (2008).

[S4] A. V. Chubukov, D. Efremov, and I. Eremin, Physical Review B 78, 134512 (2008).

[S5] K. Zantout, S. Backes, and R. Valentí, Physical Review Letters 123, 256401 (2019)

[S6] J. Fink, J. Nayak, E. Rienks, J. Bannies, S. Wurmehl, S. Aswartham, I. Morozov, R. Kappenberger, M. ElGhazali, L. Craco, et al., Physical Review B 99, 245156 (2019).

[S7] P. Dai, Reviews of Modern Physics 87, 855 (2015).

[S8] L. Fanfarillo, E. Cappelluti, C. Castellani, and L. Benfatto, Physical review letters 109, 096402 (2012).

[S9] K. Umezawa, Y. Li, H. Miao, K. Nakayama, Z.-H. Liu, P. Richard, T. Sato, J. He, D.-M. Wang, G. Chen, et al., Physical Review Letters 108, 037002 (2012).

[S10] S. Hoshino and P. Werner, Physical Review Letters 115, 247001 (2015).

[S11] H. Miao, W. Brito, Z. Yin, R. Zhong, G. Gu, P. Johnson, M. Dean, S. Choi, G. Kotliar, W. Ku, et al., Physical Review B 98, 020502 (2018).

[S12] T.-H. Lee, A. Chubukov, H. Miao, and G. Kotliar, Physical Review Letters 121, 187003 (2018).

[S13] P. Coleman, Y. Komijani, and E. J. König, Physical Review Letters 125, 077001 (2020).

[S14] Z. Yin, K. Haule, and G. Kotliar, Nature materials 10, 932 (2011).

[S15] H. Miao, P. Richard, Y. Tanaka, K. Nakayama, T. Qian, K. Umezawa, T. Sato, Y.-M. Xu, Y. Shi, N. Xu, et al., Physical Review B 85, 094506 (2012).

[S16] G. Lee, H. S. Ji, Y. Kim, C. Kim, K. Haule, G. Kotliar, B. Lee, S. Khim, K. H. Kim, K. S. Kim, et al., Physical Review Letters 109, 177001 (2012).

[S17] P. Werner, M. Casula, T. Miyake, F. Aryasetiawan, A. J. Millis, and S. Biermann, Nature Physics 8, 331 (2012).

[S18] Z. P. Yin, K. Haule, and G. Kotliar, Nature Physics 10, 845 (2014).

[S19] H. Miao, T. Qian, X. Shi, P. Richard, T. Kim, M. Hoesch, L. Xing, X.-C. Wang, C.-Q. Jin, J.-P. Hu, et al., Nature communications 6, 1 (2015).

[S20] H. Miao, Z. Yin, S. Wu, J. Li, J. Ma, B.-Q. Lv, X. Wang, T. Qian, P. Richard, L.-Y. Xing, et al., Physical Review B 94, 201109 (2016).

[S21] P. Sémon, K. Haule, and G. Kotliar, Physical Review B 95, 195115 (2017).

[S22] J. M. Tomczak, M. van Schilfgaarde, and G. Kotliar, Physical Review Letters 109, 237010 (2012).

[S23] J. Ferber, K. Foyevtsova, R. Valentí, and H. O. Jeschke, Physical Review B 85, 094505 (2012).

[S24] L. Ortenzi, E. Cappelluti, L. Benfatto, and L. Pietronero, Physical Review Letters 103, 046404 (2009).

[S25] S. Bhattacharyya, K. Björnson, K. Zantout, D. Steffensen, L. Fanfarillo, A. Kreisel, R. Valentí, B. M. Andersen, and P. Hirschfeld, arXiv preprint arXiv:2003.01638 (2020).

[S26] A. Tamai, M. Zingl, E. Rozbicki, E. Cappelli, S. Ricco, A. de la Torre, S. M. Walker, F. Bruno, P. King, W. Meevasana, et al., Physical Review X 9, 021048 (2019).

[S27] J. H. Tapp, Z. Tang, B. Lv, K. Sasmal, B. Lorenz, P. C. Chu, and A. M. Guloy, Physical Review B 78, 060505 (2008).

[S28] T. Mertz, K. Zantout, and R. Valentí, Physical Review B 98, 235105 (2018).

[S29] J. M. Tomczak, Journal of Physics: Conference Series 592, 012055 (2015).

[S30] A. L. Kutepov, V. S. Oudovenko, and G. Kotliar, Computer Physics Communications 219, 407 (2017).

[S31] A. Kutepov, K. Haule, S. Y. Savrasov, and G. Kotliar, Physical Review B 85, 155129 (2012).

[S32] A. Georges, G. Kotliar, W. Krauth, and M. J. Rozenberg, Review of Modern Physiscs 68, 13 (1996).

[S33] G. Kotliar, S. Y. Savrasov, K. Haule, V. S. Oudovenko, O. Parcollet, and C. Marianetti, Reviews of Modern Physics 78, 865 (2006).

[S34] M. Qazilbash, J. Hamlin, R. Baumbach, L. Zhang, D. J. Singh, M. Maple, and D. Basov, Nature Physics 5, 647 (2009).

[S35] L. de' Medici, G. Giovannetti, and M. Capone, Physical Review Letters 112, 177001 (2014).

[S36] S. Choi, P. Semon, B. Kang, A. Kutepov, and G. Kotliar, Computer Physics Communications 244, 277 (2019). 
[S37] P. Blaha, K. Schwarz, G. K. Madsen, D. Kvasnicka, and J. Luitz, An augmented plane wave+ local orbitals program for calculating crystal properties (2001).

[S38] P. Blaha, K. Schwarz, F. Tran, R. Laskowski, G. K. Madsen, and L. D. Marks, The Journal of Chemical Physics 152, 074101 (2020).

[S39] I. Souza, N. Marzari, and D. Vanderbilt, Physical Review B 65, 035109 (2001).

[S40] N. Marzari and D. Vanderbilt, Physical review B 56, 12847 (1997).

[S41] A. A. Mostofi, J. R. Yates, Y.-S. Lee, I. Souza, D. Vanderbilt, and N. Marzari, Computer physics communications 178, 685 (2008).

[S42] J. Kuneš, R. Arita, P. Wissgott, A. Toschi, H. Ikeda, and K. Held, Computer Physics Communications 181, 1888 (2010).

[S43] See Supplemental Material (SM) for (i) information on the construction of $H(k)$ with maximally localized Wannier function (MLWF) for DFT-LDA and LQSGW, (ii) details of the microscopic calculations include SOC, (iii) $k_{z}$ dependent electron band dispersions of $H(k)$, (iv) details of the method for the extraction of the self-energy including error bars, (v) comparison of the quality of the present LQSGW+non-local $\Sigma$ fit to the published ARPES data, showing that the fitting of the hole pockets leads to a good descriptions of the published data for $k_{z}=0.00$ [S20] and for other values of $k_{z}$. [S54], (vi) self-energy depending on the assigned $k_{z}$ value for electron pockets and corresponding Fermi surface volumes for the Luttinger's theorem, (vii) frequency dependency of the dynamical self-energy, and (viii) analysis of the transport data of Ref. [S59] and discussions of the extracted scattering rate from the transport data.

[S44] V. Brouet, D. LeBoeuf, P.-H. Lin, J. Mansart, A. Taleb-Ibrahimi, P. Le Fèvre, F. Bertran, A. Forget, and D. Colson, Physical Review B 93, 085137 (2016).

[S45] Hu Miao, unpublished.

[S46] M. Kim, J. Mravlje, M. Ferrero, O. Parcollet, and A. Georges, Physical Review Letters 120, 126401 (2018).

[S47] N.-O. Linden, M. Zingl, C. Hubig, O. Parcollet, and U. Schollwöck, Physical Review B 101, 041101 (2020).

[S48] A. Horvat, J. Mravlje, et al., Physical Review B 96, 085122 (2017).

[S49] T. Maier, M. Jarrell, T. Pruschke, and M. H. Hettler, Reviews of Modern Physics 77, 1027 (2005).

[S50] N. Qureshi, P. Steffens, Y. Drees, A. Komarek, D. Lamago, Y. Sidis, L. Harnagea, H.-J. Grafe, S. Wurmehl, B. Büchner, et al., Physical Review Letters 108, 117001 (2012).

[S51] A. Damascelli, Physica Scripta 2004, 61 (2004).

[S52] The ARPES data has been measured at $20 \mathrm{~K}$ which is slightly above the superconducting transition temperature (18 K) of LiFeAs. $\mathrm{S} 27]$.

[S53] C. Putzke, A. Coldea, I. Guillamón, D. Vignolles, A. McCollam, D. LeBoeuf, M. Watson, I. Mazin, S. Kasahara, T. Terashima, et al., Physical Review Letters 108, 047002 (2012).

[S54] Z. Wang, P. Zhang, G. Xu, L. Zeng, H. Miao, X. Xu, T. Qian, H. Weng, P. Richard, A. Fedorov, et al., Physical Review B 92, 115119 (2015).

[S55] T. Hajiri, T. Ito, R. Niwa, M. Matsunami, B. Min, Y. Kwon, and S. Kimura, Physical Review B 85, 094509 (2012).

[S56] S. Borisenko, D. Evtushinsky, Z.-H. Liu, I. Morozov, R. Kappenberger, S. Wurmehl, B. Büchner, A. Yaresko, T. Kim, M. Hoesch, et al., Nature Physics 12, 311 (2016).

[S57] R. Day, G. Levy, M. Michiardi, B. Zwartsenberg, M. Zonno, F. Ji, E. Razzoli, F. Boschini, S. Chi, R. Liang, et al., Physical Review Letters 121, 076401 (2018).

[S58] S. Choi, A. Kutepov, K. Haule, M. van Schilfgaarde, and G. Kotliar, npj Quantum Materials 1, 16001 (2016).

[S59] F. Rullier-Albenque, D. Colson, A. Forget, and H. Alloul, Physical Review Letters 109, 187005 (2012).

[S60] S. Borisenko, V. Zabolotnyy, D. Evtushinsky, T. Kim, I. Morozov, A. Yaresko, A. Kordyuk, G. Behr, A. Vasiliev, R. Follath, et al., Physical Review Letters 105, 067002 (2010).

[S61] J. Kuneš, R. Arita, P. Wissgott, A. Toschi, H. Ikeda, and K. Held, Computer Physics Communications 181, 1888 (2010).

[S62] M. J. Pitcher, D. R. Parker, P. Adamson, S. J. Herkelrath, A. T. Boothroyd, R. M. Ibberson, M. Brunelli, and S. J. Clarke, Chemical Communications , 5918 (2008).

[S63] A. Kutepov, K. Haule, S. Y. Savrasov, and G. Kotliar, 85, 155129 (2012).

[S64] A. Kutepov, V. Oudovenko, and G. Kotliar, 219, 407 (2017). 